OPEN ACCESS

Edited by:

Quan Yuan,

National Institute of Neurological

Disorders and Stroke (NINDS),

United States

Reviewed by:

Gerald Obermair,

Karl Landsteiner University of Health

Sciences, Austria

Joshua Plotkin

Stony Brook University, United States

*Correspondence:

Austen J. Milnerwood

austen.milnerwood@mcgill.ca

Specialty section:

This article was submitted to

Cellular Neurophysiology,

a section of the journal

Frontiers in Cellular Neuroscience

Received: 02 June 2020 Accepted: 26 January 2021 Published: 18 February 2021

Citation: Kuhlmann N, Wagner Valladolid M, Quesada-Ramirez L, Farrer MJ and Milnerwood AJ (2021) Chronic and Acute Manipulation of Cortical

Glutamate Transmission Induces Structural and Synaptic Changes in

Co-cultured Striatal Neurons.

Front. Cell. Neurosci. 15:569031. doi: 10.3389/fncel.2021.569031

\section{Chronic and Acute Manipulation of Cortical Glutamate Transmission Induces Structural and Synaptic Changes in Co-cultured Striatal Neurons}

\author{
Naila Kuhlmann ${ }^{1,2}$, Miriam Wagner Valladolid ${ }^{1}$, Lucia Quesada-Ramírez ${ }^{1}$, \\ Matthew J. Farrer ${ }^{1,3}$ and Austen J. Milnerwood ${ }^{1,2 *}$
}

${ }^{1}$ Centre for Applied Neurogenetics (CAN), University of British Columbia, Vancouver, BC, Canada, ${ }^{2}$ Montreal Neurological Institute, Department of Neurology and Neurosurgery, McGill University, Montreal, QC, Canada, ${ }^{3}$ McKnight Brain Institute, University of Florida, Gainesville, FL, United States

In contrast to the prenatal topographic development of sensory cortices, striatal circuit organization is slow and requires the functional maturation of cortical and thalamic excitatory inputs throughout the first postnatal month. While mechanisms regulating synapse development and plasticity are quite well described at excitatory synapses of glutamatergic neurons in the neocortex, comparatively little is known of how this translates to glutamate synapses onto GABAergic neurons in the striatum. Here we investigate excitatory striatal synapse plasticity in an in vitro system, where glutamate can be studied in isolation from dopamine and other neuromodulators. We examined pre-and post-synaptic structural and functional plasticity in GABAergic striatal spiny projection neurons (SPNs), co-cultured with glutamatergic cortical neurons. After synapse formation, medium-term (24 h) TTX silencing increased the density of filopodia, and modestly decreased dendritic spine density, when assayed at 21 days in vitro (DIV). Spine reductions appeared to require residual spontaneous activation of ionotropic glutamate receptors. Conversely, chronic (14 days) TTX silencing markedly reduced spine density without any observed increase in filopodia density. Time-dependent, biphasic changes to the presynaptic marker Synapsin-1 were also observed, independent of residual spontaneous activity. Acute silencing $(3 \mathrm{~h})$ did not affect presynaptic markers or postsynaptic structures. To induce rapid, activity-dependent plasticity in striatal neurons, a chemical NMDA receptor-dependent "long-term potentiation (LTP)" paradigm was employed. Within $30 \mathrm{~min}$, this increased spine and GluA1 cluster densities, and the

\footnotetext{
Abbreviations: SPN, striatal projection neuron; D1R, Drd1 dopamine receptor; D2R, Drd2 dopamine receptor; LTP, long-term potentiation; LTD, long-term depression; TTX, tetrodotoxin; DIV, days in vitro; AP5, D-(-)-2-Amino-5phosphonopentanoic acid; CNQX, 6-cyano-7-nitroquinoxaline-2, 3-dione disodium salt; mEPSC, miniature excitatory post-synaptic current; cLTP, chemical LTP; PTX, picrotoxin; NGS, normal goat serum; PBS, phosphate-buffered saline; RT, room temperature.
} 
percentage of spines containing GluA1 clusters, without altering the presynaptic signal. The results demonstrate that the growth and pruning of dendritic protrusions is an active process, requiring glutamate receptor activity in striatal projection neurons. Furthermore, NMDA receptor activation is sufficient to drive glutamatergic structural plasticity in SPNs, in the absence of dopamine or other neuromodulators.

Keywords: cortico-striatal co-culture, immunocytochemistry, electrophysiology, dendritic spines, synaptic plasticity, glutamate, long-term potentiation

\section{INTRODUCTION}

The striatum is a highly integrative structure. In rodents, each of the $\sim 2.5$ million GABAergic medium-sized spiny projection neurons (SPNs) receives $\sim 25,000$ glutamate afferents from nearly all areas of the cortex and thalamus (Kincaid et al., 1998; Doig et al., 2010). These are modulated by nigrostriatal dopamine and form the only striatal output pathways (Tritsch and Sabatini, 2012). As the gateway to the basal ganglia, the striatum mediates action selection, motor control, motivation, and learning (Friend and Kravitz, 2014), and its dysfunction is implicated in neurodevelopmental disorders, addiction, and neurodegeneration (Graybiel et al., 2000; Smith et al., 2009; Gerfen and Surmeier, 2011). In contrast to the prenatal topographic development of sensory cortices, striatal circuit organization is slow and requires the functional maturation of cortical and thalamic excitatory inputs throughout the first postnatal month (Tepper et al., 1998). During this time, glutamate release promotes the formation and stabilization of excitatory synapses on SPNs (Kozorovitskiy et al., 2012, 2015). While mechanisms regulating synaptic development, maintenance, and plasticity are quite well described at glutamate synapses in principal excitatory neurons of the neocortex, much less is known of how these mechanisms translate to subcortical areas such as the striatum. Here, we developed assays to investigate excitatory synapse plasticity in a striatal in vitro system, where glutamate activity can be examined in isolation from dopamine and other neuromodulators.

Dendritic spines are specialized excitatory post-synaptic structures, which are thought to compartmentalize signaling processes to regulate glutamate receptor activation, calcium flux, cytoskeletal remodeling, membrane trafficking, and protein synthesis/degradation (Bourne and Harris, 2008; Yoshihara et al., 2009). Activity-dependent morphological changes in dendritic spines and associated presynaptic elements modulate neural function, with growth, pruning, and remodeling likely underlying cognitive processes (Villalba and Smith, 2013; Sala and Segal, 2014), and spine loss being a potential structural correlate of cognitive deficits (Penzes et al., 2011).

Excitatory synapse development, spine formation, and dynamics have been extensively studied in hippocampal/cortical pyramidal neurons, both in vivo and in vitro. Long-term potentiation (LTP) and long-term depression (LTD)-like paradigms have received particular attention, as these lasting activity-dependent modifications are considered the leading cellular model for learning and memory (Bliss and Collingridge,
1993). Spines alter shape and receptor composition in response to plasticity induction paradigms, with studies showing that LTP is typically associated with spine swelling (Matsuzaki et al., 2004; Okamoto et al., 2004; Tanaka et al., 2008) or de novo spine formation (Maletic-Savatic and Malinow, 1998; Engert and Bonhoeffer, 1999; Goldin et al., 2001), whereas LTD is associated with spine shrinkage (Okamoto et al., 2004; Zhou et al., 2004) or spine loss (Nägerl et al., 2004).

In recent genetic models where glutamate transmission is absent, principle excitatory neurons still develop normal dendritic architecture and spine numbers in vivo (Sando et al., 2017), a recent finding that adds weight to the traditional view that neuronal activity serves as a mechanism of refinement after synaptic connections are established (LeVay et al., 1980; Katz and Shatz, 1996; Sanes and Lichtman, 1999; Huberman et al., 2008). Conversely, there is evidence to support that activity is also important for synapse formation per se (Sabo et al., 2006; Andreae and Burrone, 2014; Choi B. J. et al., 2014; Choi S. H. et al., 2014; Okawa et al., 2014), reviewed in Andreae and Burrone (2018). In neuronal cultures, chronic action potential silencing throughout synaptogenesis reduces spine density by $\sim 50 \%$ (Kossel et al., 1997), whereas after synapses form, a 3-day (but not $24 \mathrm{~h}$ ) silencing period reduces spine number by only $\sim 15 \%$ (Papa and Segal, 1996), suggesting that activity throughout the first 3 days contributes to synapse (and consequently spine) formation.

Alternatively, the observations that spine formation occurs on pyramidal neurons in the absence of vesicular glutamate release (Sando et al., 2017; Sigler et al., 2017), but is reduced in chronically-silenced cultures (Kossel et al., 1997), raise the possibility that early stages of spine formation may be regulated by GABAergic activity; inhibitory neurons are present in both scenarios, and GABA transmission (which is depolarizing in early development, and thus excitatory), is blocked in silenced cultures. Against this suggestion as a general rule, striatal cultures (almost entirely comprised of GABAergic SPNs) fail to generate appropriate dendritic arbors or dendritic spines in the absence of glutamatergic neurons, but do when co-cultured with cortical or hippocampal neurons (Segal et al., 2003; Kaufman et al., 2012; Fasano et al., 2013; Paraskevopoulou et al., 2019). Chronic silencing prevents spine formation even on co-cultured SPNs, which will develop spines within $2 \mathrm{~h}$ of TTX wash-out (Segal et al., 2003); this suggests connections are made despite silencing, and that spinogenesis specifically requires action potential-dependent transmission. Thus, SPNs require glutamatergic input to develop their 
eponymous morphology, and their dendritic spines appear to be highly plastic.

Alterations to striatal dendritic structures and synaptic plasticity are observed in multiple disorders, with evidence from many studies suggesting a role in the pathophysiology of Parkinson's disease, Alzheimer's disease, schizophrenia, and autism (McNeill et al., 1988; Day et al., 2006; Milnerwood and Raymond, 2010; Penzes et al., 2011; Villalba and Smith, 2013; Sala and Segal, 2014; Volta et al., 2017). Most knowledge of striatal plasticity comes from electrophysiological studies in acute brain slices; these have demonstrated a propensity to presynaptic plasticity and long-term depression, as well as the importance of neuromodulation by dopamine (Calabresi et al., 1997; Spencer and Murphy, 2000; Wang et al., 2006; Kreitzer and Malenka, 2007; Sergeeva et al., 2007; Li et al., 2009; Lovinger, 2010; Blackwell et al., 2019). That said, glutamate uncaging has also been shown to be sufficient to trigger de novo spine formation on SPN dendrites in $\sim 50 \%$ of trials (Kozorovitskiy et al., 2012, 2015), similar to basal rates in cortical pyramidal neurons (Kwon and Sabatini, 2011). Elsewhere, Shen et al. (2008) demonstrated that dopamine was necessary for determining the directionality, but not necessarily the induction, of spike-time dependant plasticity in cultured striatal slices. Thus, glutamatergic modulation of striatal dendritic spines merits further attention, both during development and in response to activity-dependant plasticity.

Here, we examined how glutamate transmission, in the absence of dopamine, modulates SPN dendritic spine development, plasticity, and associated synaptic markers. Using an in vitro cortico-striatal co-culture system (Segal et al., 2003; Tian et al., 2010; Randall et al., 2011; Kaufman et al., 2012; Milnerwood et al., 2012; Penrod et al., 2015), we investigated the effects of blocking action potential-dependent network activity, manipulating spontaneous AMPA receptor (AMPAR) and NMDA receptor (NMDAR) activity, and the effects of an NMDAR-dependent LTP induction paradigm. Long-term glutamate silencing $(>24 \mathrm{~h})$ induced presynaptic alterations, reduced spine density, and had variable effects on filopodia, while short-term silencing $(<3 \mathrm{~h})$ did not. The LTP induction paradigm rapidly induced spine and GluAl cluster changes, consistent with LTP-like modifications. We add to the literature by showing that glutamatergic activity is required for the maturation of striatal neurons, and demonstrate that glutamate receptor activity can induce structural plasticity in the absence of dopamine or other neuromodulators. The experiments here provide a foundation for future studies of activity-dependent striatal plasticity both in development and disease.

\section{MATERIALS AND METHODS}

\section{Culture Preparation}

Wild-type (WT) C57BL/6J, and WT littermates from an LRRK2 G2019S knock-in colony (bred with the C57BL/6 colony, described in Beccano-Kelly et al., 2014) were maintained following the University of British Columbia animal care unit and the Canadian Council on Animal Care regulations. Primary neuronal cultures were prepared from mouse embryos (E16.5) of either sex. Briefly, brains were removed and dissected on ice in Hank's Balanced Salt Solution (HBSS, GIBCO). For WT littermate cultures, tails were genotyped before cells were pooled, as in Beccano-Kelly et al. (2014). Cortical and striatal tissues were separately digested in $0.05 \%$ Trypsin-EDTA (LifeTech) at $37^{\circ} \mathrm{C}$. Striatal cells were nucleofected with GFP on an AAV plasmid driven by a long-lasting $(\mathrm{CAG} / \beta$-actin) promoter (pAAV-CAG-GFP; Addgene plasmid \#37825): 1-2 million cells were suspended in $100 \mu \mathrm{l}$ of electroporation buffer (Mirus Bio) with 1-2 $\mu \mathrm{g}$ of endonuclease-free DNA, transferred to a cuvette and electroporated using a Lonza Nucleofector 2b (Amaxa, program 05). The cell suspension was then removed and resuspended in plating medium (PM; 2\% B27 + $1 / 100$ penicillin/streptomycin, Invitrogen; $0.5 \mathrm{mM} \alpha$-glutamine; neurobasal medium, GIBCO) and 24-well plates were seeded with non-transfected cortical neurons from the same mice at $1: 1$, to a density of 200,000 cells/well in $1 \mathrm{ml}$ of PM. Cells were incubated at $37^{\circ} \mathrm{C}$ and $5 \% \mathrm{CO}_{2}$, and, from days in vitro (DIV) 4 onwards, $10 \%$ of media was exchanged every 3-5 days until DIV21.

To verify survival and correct fluorophore/morphological identification of SPNs (as opposed to striatal interneurons and other cells) in vitro, additional co-cultures were prepared from homozygous BAC transgenic Drd1a-tomato mice [D1RTom, B6.Cg-Tg(Drd1a-tdTomato)6Calak/J, Jackson Laboratory, \#016204], in which SPNs expressing the Drd1 dopamine receptor (D1R) are identified by Td-Tomato red fluorescent protein (Ade et al., 2011). To visualize SPNs expressing the Drd2 dopamine receptor (D2R), we used heterozygous Drd2-eGFP transgenic mice on an FVB/NJ background (D2R-eGFP, a gift from Raymond lab). Striatal or cortical neurons were nucleofected with TagBFP (pTagBFP-N; Axxora; EVN-FP172-C020) before plating 1:1 with non-nucleofected cells, and maintained until DIV21 as described above.

\section{Treatments}

\section{Chronic and Acute Action Potential Silencing}

Co-cultures were left untreated 7 days after plating, to enable neurite outgrowth and synapse formation. Action potentials were then blocked by TTX application [ $1 \mu \mathrm{M}$; Tocris $\left(\mathrm{IC}_{50} \sim 7 \mathrm{nM}\right.$ )] in two ways: (A) throughout the rest of the 3-week development and maturation process, $3 \times$ TTX (TTX added at DIV7, 14 and 20 ); and (B) for the first 2 weeks with no further addition within the third week, $2 \times$ TTX (TTX at DIV7 and 14), and compared to control (sham; no drug added) neurons on the same 24-well plate. The concentration (far exceeding $\mathrm{IC}_{50}$ ) and time of TTX application was chosen to ensure effectiveness with fresh media addition (Takada et al., 2005; Hartman et al., 2006; Fishbein and Segal, 2011), and the $2 \times$ TTX (B) group included to see if a 7 -day period is sufficient for recovery. One-hundred microliter of media was removed from each well and pooled by condition, then returned to each well, with, or without (sham) the addition of TTX.

Short-term disruption of glutamate signaling was achieved by blockade of burst firing with TTX (1 $\mu \mathrm{M}$; Silencing), or of all excitatory activity (Total Silencing) by application of TTX, 6-cyano-7-nitroquinoxaline-2,3-dione disodium salt 
(CNQX; AMPA/kainite receptor antagonist; $10 \mu \mathrm{M}$; Tocris) and D-(-)-2-Amino-5-phosphonopentanoic acid (AP5; NMDA receptor antagonist; $10 \mu \mathrm{M}$; Tocris). At 24 or $3 \mathrm{~h}$ before fixation on DIV21, $100 \mu \mathrm{l}$ of media was removed from each well and pooled by condition, then replaced with (for silencing) or without (sham control) drug addition.

\section{Chemical Plasticity}

The chemical long-term potentiation (cLTP) paradigm was achieved by applying glycine in the absence of extracellular magnesium $\left(\mathrm{Mg}^{2+}\right)$, as previously described in hippocampal neurons (Lu et al., 2001; Brigidi et al., 2014). Briefly, media was removed from wells and replaced by an $\mathrm{Mg}^{2+}$-free extracellular solution (ECS; $125 \mathrm{mM} \mathrm{NaCl}, 33 \mathrm{mM}$ D-glucose, $5 \mathrm{mM}$ HEPES, $5 \mathrm{mM} \mathrm{KCl}, 2 \mathrm{mM} \mathrm{CaCl} 2$ ) containing $0.5 \mu \mathrm{M}$ TTX and $20 \mu \mathrm{M}$ bicuculline methiodide (10 mM stock; Tocris) for $15 \mathrm{~min}$. Onehundred microliter of the solution was then removed from each well, $200 \mu \mathrm{M}$ glycine (100 mM stock; Thermo Fisher Scientific) was added for cLTP condition and the solution was replaced, whereas removal/replacement without glycine addition acted as a negative sham control (cLTP Control). After $3 \mathrm{~min}$, the solution in both groups was replaced with a fresh solution for $30 \mathrm{~min}$, before fixation. A media removal and replacement group (without a change to $\mathrm{Mg}^{2+}$-free) acted as a second sham control.

\section{Immunostaining}

Cells were fixed [4\% Paraformaldehyde (PFA), 4\% sucrose; $20 \mathrm{~min}]$, permeabilized $\left[-20^{\circ} \mathrm{C}\right.$ Methanol $(\mathrm{MeOH})$ for $\left.3 \mathrm{~min}\right]$ and blocked $[3 \times 20$ min wash with $10 \%$ normal goat serum (NGS) in phosphate-buffered saline (PBS), at room temperature (RT)]. Primary antibodies were incubated by shaking overnight at $4^{\circ} \mathrm{C}$ in PBS with Tween 20 (PBST) $+2 \%$ NGS, then cells were blocked again (10\% NGS + PBS, 1 h RT) before secondary antibodies were applied (in PBST + 2\% NGS, 30 min RT). Coverslips were washed (PBS, $3 \times 10 \mathrm{~min}$ ) and slide-mounted with Fluoromount (Southern Biotech). The primary antibodies used were anti-GFP (Green Fluorescent Protein, mouse, Abcam Cat\# ab1218 RRID: AB_298911, 1:1,000), anti-synapsin1 (Synapsin-1, rabbit, Millipore Cat\# AB1543P RRID: AB_90757, 1:500), anti-GluA1 (AMPA Receptor, rabbit, Alomone Labs Cat\# AGC-004 RRID: AB_2039878, 1:500), anti-tRFP (tagRFP, rabbit, Axxora Cat\# EVN-AB233, 1:500). Secondary antibodies were anti-Mouse Alexa 488 (RRID: AB_2534069), anti-Rabbit Alexa 568 (RRID: AB_143157) and anti-Rabbit AMCA (all 1:1,000).

\section{Image Acquisition and Quantification}

For co-culture characterization, 10-15 images were captured of each culture on an Olympus Fluoview 1000 confocal microscope (20× magnification, $1 \times$ confocal zoom), at random points across coverslips (for striatal marker co-expression counts) and targeted at BFP-expressing neurons (to verify D1R or D2R co-expression specifically with nucleofected neurons). The number of nucleofected neurons (blue) co-expressing Td-Tomato (red, D1R) or eGFP (green, D2R) were counted in ImageJ.

For other experiments, GFP-expressing neurons that fit D1R or D2R SPN morphology (Kaufman et al., 2012) were imaged as a series of $8-15$ successive $0.5 \mu \mathrm{m} z$-stacks $(60 \times$ oil immersion lens, $2 \times$ confocal zoom). Five to 10 SPNs were imaged per condition from a minimum of three independent cultures, with excitation and acquisition parameters constrained across all paired comparisons. The acquired images were sorted by channel and flattened using the max projection function on ImageJ for dendritic protrusion and cluster analysis.

For GluA1 and Synapsin-1 puncta analysis, images were manually thresholded and binarized by the eye using ImageJ, with the experimenter blind to condition. All quantification was conducted in Cell Profiler (http://www.cellprofiler.org; analysis pipeline included in Supplementary Material). Briefly, GFP-expressing cells were used to mask the dendritic arbor as the region of interest (ROI), which was then expanded by five pixels to capture apposing presynaptic elements. Binarized Synapsin-1 or GluA1 images were used to produce masks within the dendritic ROI, which was applied to the corresponding original (non-binarized) image to obtain puncta size (min diameter $=4$ pixels; $\max =15$ pixels), intensity, and density (number of puncta/dendrite length) measures. Otsu's method was used for automatic global thresholding of the images, and adjacent puncta were distinguished and divided by intensity.

To quantify dendritic protrusions, $3 \times \geq 30 \mu \mathrm{m}$ segments of secondary or tertiary dendrites, at least $30 \mu \mathrm{m}$ from the soma were selected in the green channel (GFP fill) of each $z$-projected image in ImageJ. Dendrite length was recorded and manual 2D digital reconstruction was performed to count and measure each dendritic protrusion, with the experimenter blind to treatment condition. Protrusions were classified as either spine $(<2 \mu \mathrm{m}$ in length with a visible head $>0.5 \mu \mathrm{m}$ in diameter), or filopodia if they ranged between $1-10 \mu \mathrm{m}$ and lacked a distinct bulbous head (Segal et al., 2003; Arstikaitis et al., 2011). The calculated densities and lengths for individual dendrites were averaged for a mean density per neuron. To quantify the percentage of spines associated with GluA1, clusters from binarized GluA1 images were manually counted within spines in three selected dendritic segments, excluding clusters that were clearly in perpendicular crossing neurites of other neurons.

Additional analysis was conducted on a large subset of images from chronic and acute silencing experiments, to quantify Synapsin-1 puncta on excitatory synapses only (those on spines and filopodia-like protrusions), as opposed to the entire dendrite masks. Image J was used to create ROIs around a sample of 20 spines (of varying shapes and widths) and any visible filopodia (ranging from 0 to 20) on secondary or tertiary dendrites of the GFP-expressing cell in each image. The ROIs were then applied as masks on the corresponding raw Synapsin-1 images, and the mean and integrated intensity measured within each ROI.

\section{Electrophysiology}

Whole-cell voltage-clamp recordings were performed on GFP-expressing SPNs in the cortico-striatal co-cultures at DIV20-22 to measure functional changes following glycine application. $30 \mathrm{~min}$ after the cLTP or cLTP Control treatment, cells were perfused at room temperature with the extracellular solution (ECS) containing (in $\mathrm{mM}$ ): 167 sodium chloride, 2.4 potassium chloride, one magnesium chloride, 10 glucose, 10 HEPES, two calcium chloride; pH 7.4, 290-300 mOsm. TTX 
$(1 \mu \mathrm{M})$ and picrotoxin (PTX, $100 \mu \mathrm{M})$ were added to block spontaneous burst firing and GABAergic activity respectively. Pipette resistance ( $\mathrm{Rp}$ ) was 5-8 $\mathrm{M} \Omega$ when filled with (in $\mathrm{mM}$ ): 130 cesium methanesulfonate, five cesium chloride, four sodium chloride, one magnesium chloride, 5 EGTA, 10 HEPES, 5 QX-314, 0.5 GTP, 10 Na2-phosphocreatine, and 5 Mg ATP, 0.1 spermine; $\mathrm{pH} 7.3,290 \mathrm{mOsm}$. The membrane test function was used to determine intrinsic membrane properties after obtaining whole-cell configuration, with a holding potential of $-70 \mathrm{mV}$ (Milnerwood et al., 2012). Following a 2-min settling period, miniature (spontaneously released, in the presence of TTX) excitatory post-synaptic currents (mEPSCs) were recorded at $-70 \mathrm{mV}$. Data were acquired by Multiclamp $700 \mathrm{~B}$ amplifier and signals were filtered at $2 \mathrm{kHz}$, digitized at $10 \mathrm{kHz}$, and analyzed in Clampfit10 (Molecular Devices). Only recordings with a series resistance (Rs) $<30 \mathrm{M} \Omega$ were included and $\Delta \mathrm{Rs}$ tolerance cut-off was $<10 \%$. mEPSCs were analyzed using the threshold search in Clampfit10 (threshold $5 \mathrm{pA}$ ) and additional visual quality control with the experimenter blind to genotype; monophasic events were used for amplitude and decay kinetics, while others were suppressed but included in frequency counts.

\section{Statistical Analysis}

All statistical analyses were performed using Graphpad versions 7-9 (GraphPad software). For chronic and acute TTX experiments, spine/filopodia analysis is presented as raw data, whereas Synapsin-1 cluster data is normalized to the sham control within culture, to account for betweenculture variation in immunostaining. Analyses were performed by one-way ANOVA and a Kruskal-Wallis test when data were not normally distributed (based on the d'Agostino and Pearson omnibus normality test). If significance was reached (at $p<0.05$ ), post-hoc comparisons were made using uncorrected Fisher's LSD (following one-way ANOVA) or uncorrected Dunn's test (following Kruskal-Wallis). For chemical plasticity, the cLTP condition was normalized to cLTP control within culture, and comparisons made using two-tailed unpaired Student's $t$-test, or the Mann-Whitney $U$ test when data were not normally distributed. Statistical analyses are specified in each figure legend and all significant comparisons displayed by asterisks, with sample numbers $(n)$ presented as number of images (number of independent cultures). Data are presented as mean \pm SEM throughout.

\section{RESULTS}

\section{Characterization of Cortico-Striatal Co-cultures From D1R and D2R Reporter Mice}

While striatal neurons develop poorly and have low viability in mono-culture (Segal et al., 2003; Kaufman et al., 2012; Burguière et al., 2013), those co-cultured with cortical neurons develop complex dendritic arbors and spines that stabilize around DIV20, and exhibit both morphological and electrophysiological properties resembling SPNs in vivo (Segal et al., 2003; Tian et al., 2010; Randall et al., 2011; Kaufman et al., 2012; Milnerwood et al.,
2012; Burguière et al., 2013; Lalchandani et al., 2013; Penrod et al., 2015). Over 95\% of total striatal cells are SPNs in vivo (Kawaguchi and Kubota, 1997), a proportion that is maintained in vitro (Shehadeh et al., 2006). Of these, half express the D1 dopamine receptor, and half express the D2 dopamine receptor (Kreitzer, 2009).

To verify the nucleofection of isolated striatal cells before mixing in co-culture, and ensure correct visual identification of SPNs by the experimenter based on fluorescent fills, we quantified the co-expression of BFP plasmid-nucleofected striatal neurons in cultures prepared from germ-line SPN marker mice; for D1R SPNs we used Drd1a-tdTomato reporter mice, and for D2R SPNs we used Drd2-eGFP reporter mice. BFP-expressing neurons showing characteristic SPN morphology, as described in previous studies (Kaufman et al., 2012; Burguière et al., 2013), were imaged before checking for D1R or D2R co-expression, to test the accuracy of the experimental assessment. Given that $>95 \%$ of striatal cells are SPNs, D1- and D2-expressing cells should each account for $\sim 50 \%$ of all BFP-expressing neurons; however, it should be noted that in acute slices and cultures, the segregation in double-fluorophore mice is $60 \% \mathrm{D} 1$ vs. $40 \% \mathrm{D} 2$ (Thibault et al., 2013). In line with this, $56 \%$ of imaged BFP neurons (in two separate cultures) in cultures from Drd1a-tdTomato reporter mice were Drd1 positive (Figures 1A,B). In cultures from Drd2-eGFP reporter mice (Figure 1C), $\sim 28 \%$ of BFP-expressing neurons (over two separate cultures) co-expressed D2R, slightly below the expected proportion. Together the results demonstrate that at least $80 \%$ of BFP-filled cells are clearly identified as SPNs, based on BAC fluorophore expression.

\section{Chronic TTX Application During Development Alters Dendritic Protrusions and Synapsin-1 Clusters in SPNs}

The role of bursts of synchronous (action potential-mediated) excitatory release onto SPNs during synapse maturation was assessed by chronic blockade of action potentials (TTX) in cortico-striatal co-cultures over $2(2 \times$ TTX, at DIV 7,14$)$ or 3 $(3 \times$ TTX, at DIV 7, 14 and 20) weeks (Figure 2A). Quantification of dendritic protrusions on GFP-expressing SPNs (Figure 2B) revealed a significant effect of treatment upon SPN spines (Figure 2Ci), with post-hoc analysis demonstrating significantly lower density in both TTX-treated groups, relative to untreated SPNs (control $=0.68 \pm 0.07,2 \times$ TTX $=0.46 \pm 0.05$, and $3 \times$ TTX $=0.36 \pm 0.04$ spines $/ \mu \mathrm{m}$ dendrite). While filopodia density was not significantly altered, there was a clear trend toward TTX treatment increasing filopodia in a dose/timedependent manner (Figure 2Cii).

To assess whether postsynaptic structural alterations were associated with a change in presynaptic contacts, we quantified Synapsin-1 clusters (present at both glutamatergic and GABAergic synapses) in contact with dendrites on GFP-filled SPNs. The density of Synapsin-1 clusters did not differ between treatment groups (Supplementary Figure 1A), but there was a significant main effect of treatment upon cluster size (Figure 2Di) and cluster intensity (Figure 2Dii), 

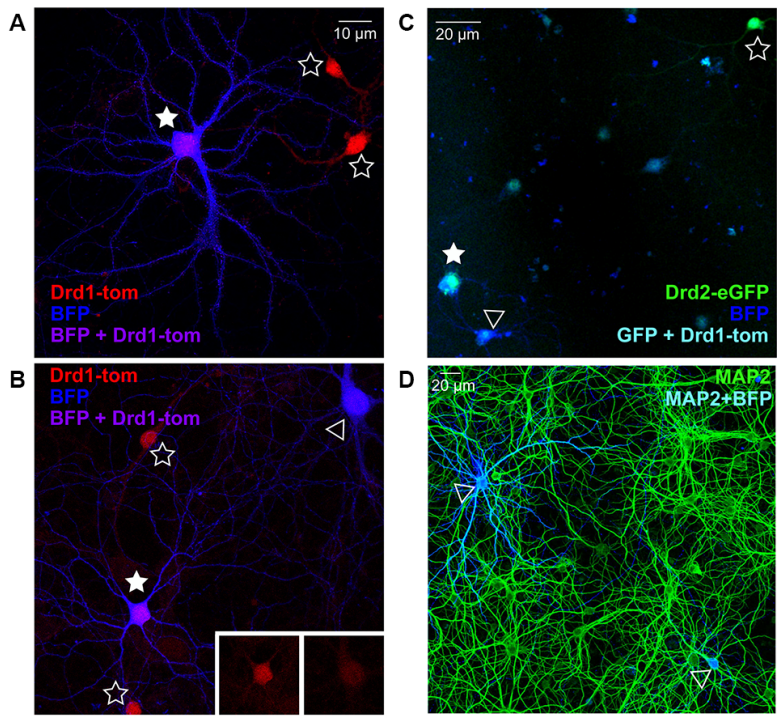

FIGURE 1 | Nucleofection and spiny projection neuron (SPN) identification in co-cultures from reporter line mice. $(\mathbf{A}, \mathbf{B})$ Representative images of days in vitro (DIV) 21 striatal neurons from Drd1a-tdTomato (D1R) BAC transgenic reporter line mice, nucleofected with BFP expression constructs and grown in co-culture with cortical neurons (20x magnification, $2 \times$ zoom Olympus FV-1000). (A) D1R SPN (red) co-labeled with BFP-fill (blue) is shown (purple; filled star), surrounded by two non-nucleofected (open star) D1R SPNs. (B) Two BFP filled SPNs are apparent, one of which is D1R+ve (filled star) and one is negative (open arrowhead), as apparent in somatic D1 signal (inserts); two D1R+ve non-nucleofected SPNs are nearby (open stars). (C)

Representative image of DIV21 striatal neurons from Drd2-eGFP (D2R) BAC transgenic reporter line mice, nucleofected with BFP expression constructs and grown in co-culture with cortical neurons $(20 \times$ magnification, Olympus FV-1000). A D2R SPN (green) co-labeled with BFP-fill (blue) is shown (cyan; filled star), near to a non-nucleofected (open star) D2R SPN. A BFP filled SPN is apparent, which is D2R+ve (open arrowhead). (D) Representative image (20x magnification) of DIV21 cortico-striatal co-cultures from non-transgenic mice. Striatal neurons nucelofected with BFP expression constructs (blue) before plating, and cultures were stained for MAP2 (green) to verify the density of nucleofected striatal neurons. Two BFP-expressing neurons are visible (open arrowheads)

with both TTX-treated groups showing a reduction when normalized to control SPN values $(2 \times$ TTX $=0.85 \pm 0.05$ and $3 \times \mathrm{TTX}=0.87 \pm 0.16)$. The size of Synapsin- 1 clusters was only significantly reduced compared to control SPNs in the $2 \times$ TTX condition (Figure 2Di, $0.93 \pm 0.02$ ). To verify that the observed changes occurred at excitatory synapses, we quantified puncta signal intensity directly on spines and filopodia (Supplementary Figure 1A); in agreement with reduced presynaptic intensity on whole dendritic masks, Synapsin-1 signal was significantly reduced on spines in cultures that were silenced for the full period $(3 \times$ TTX), relative to both untreated and transiently-silenced cultures $(2 \times$ TTX, $p<0.0001$ and $p=0.008$, respectively). While not statistically significant, there was an intermediate reduction in Synapsin-1 signal on spines in the transientlysilenced group. This demonstrates that presynaptic alterations in Synapsin-1 signal on spines (Supplementary Figure 1A) correlate with the reductions to spine density (Figure 2Ci) and Synapsin-1 signal on whole dendrite masks (Figure 2D).
Silencing duration gradually increased the density of filopodia, but not significantly (Figure 2Cii), and while Synapsin-1 signal was unaltered on filopodia of cultured SPNs silenced for the full period, in the transiently-silenced group Synapsin-1 signal was significantly increased, relative to both untreated and total silenced (Supplementary Figure 1A). This suggests that the overall reduction in presynaptic signals onto SPN dendrites of silenced cultures is predominantly at more mature dendritic spines and that transient silencing results in an increase in presynaptic signal on filopodia (and a rebound increase in spines) following removal of TTX.

Together the data demonstrate that sustained chronic blockade of burst firing in cortico-striatal co-cultures alters presynaptic inputs, in concert reducing the density of dendritic spines by either: (1) preventing spine formation in SPNs (which recovers partly when TTX is removed); or (2) causing a gradual loss of spines that is more pronounced with longer silencing.

\section{Glutamatergic Silencing Alters Dendritic Protrusions and Synapsin-1 Clusters After 24, but Not 3, Hours}

Next, we tested whether pre-and post-synaptic changes would still be observed following a shorter $(24 \mathrm{~h})$ TTX application (Silencing), and after additionally using antagonists to block glutamate signaling from action potential-independent (miniature/spontaneous) release and AMPA and NMDA receptor signaling (Total Silencing, Figure 3A). A treatment effect on spine density in GFP-filled SPNs (Figure 3B) neared statistical significance (Figure $\mathbf{3 C i}, p=0.07$ ), due to reduced spine density in the TTX Silencing group, whereas Total silencing appeared to prevent this. There was a significant main effect of treatment upon filopodia density, with a significant increase following TTX application only, and a strong trend to an increase following total silencing (Figure 3Cii).

Analysis of Synapsin-1 revealed that cluster density was not altered (Supplementary Figure 1B), but cluster size was significantly increased in both the Silencing and Total Silencing conditions when normalized to control SPNs (Figure 3Ciii, $1.08 \pm 0.01, p<0.0001$ and $1.10 \pm 0.02$, respectively) Cluster intensity was also significantly increased after Total Silencing, whereas there was only a trend to increase in the Silencing condition (Figure 3Civ). Analysis of Synapsin-1 signal specifically on dendritic spines and filopodia demonstrated an increase in spines similar to that observed on whole dendrite masks, but no change on filopodia (Supplementary Figure 1B). The data suggest presynaptic alterations (increased Synapsin-1 signal) occur on all spines upon TTX silencing, before robust spine elimination by longer (chronic) TTX silencing; this spine loss is prevented by blocking residual spontaneous activity in the Total Silencing group, despite similar presynaptic effects. Conversely, no presynaptic changes were detected on filopodia, despite their increased density, which must require more sustained (chronic) silencing.

A shorter $3 \mathrm{~h}$ blockade of glutamatergic activity produced no significant differences in spine density (Figure 3Di), filopodia 
A

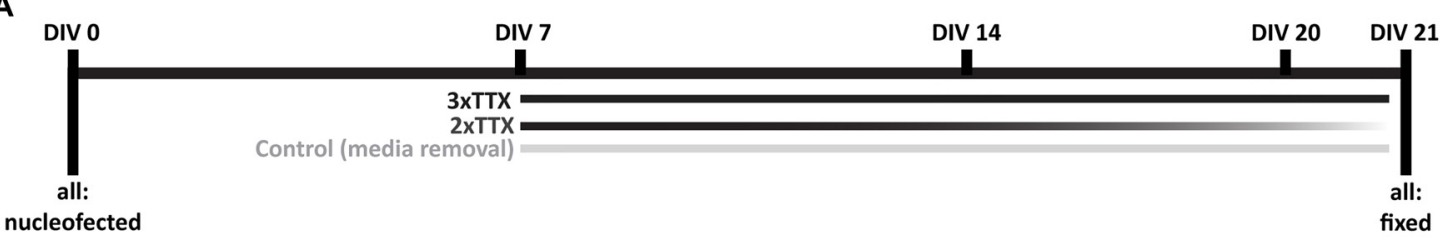

B Control
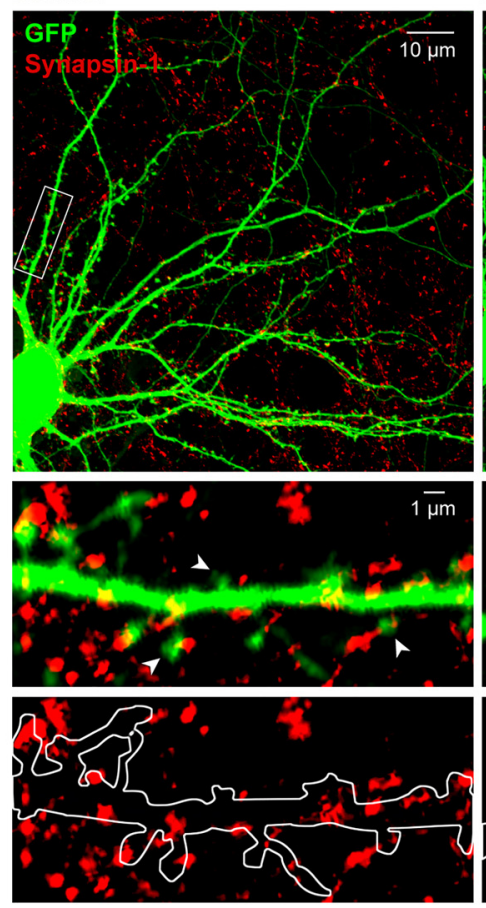

c i.

Spines

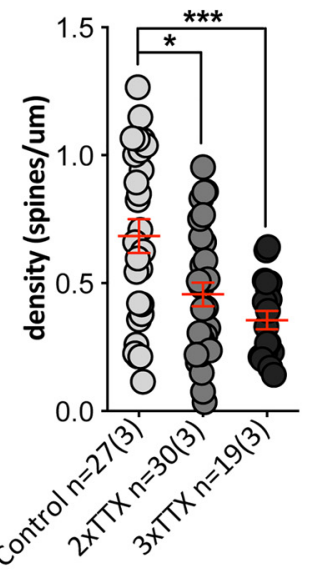

ii.
2xTTX (DIV 7,14)
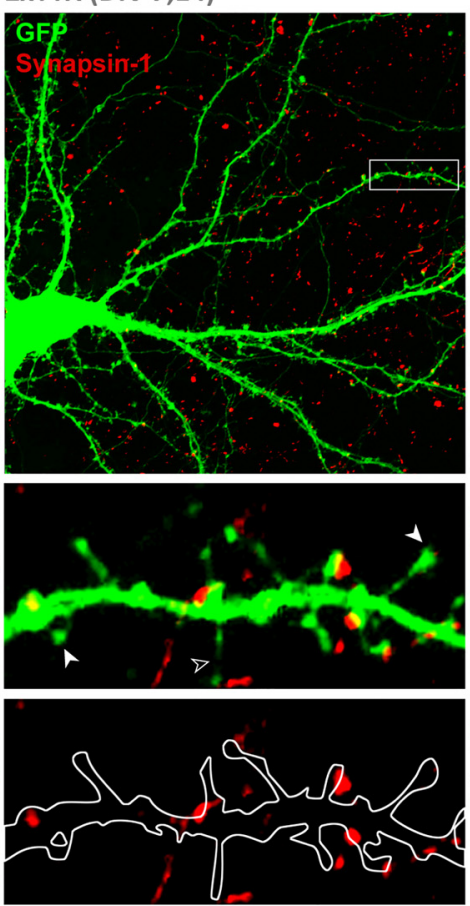

Filopodia

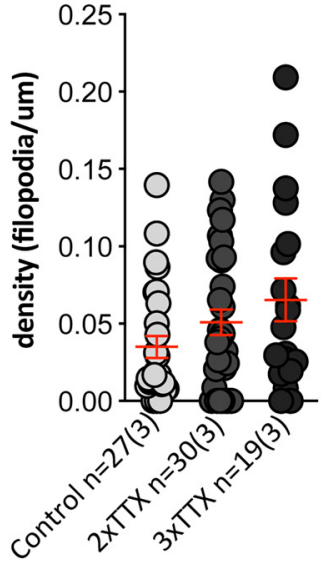

D i.

Synapsin-1
3xTTX (DIV 7,14,20)
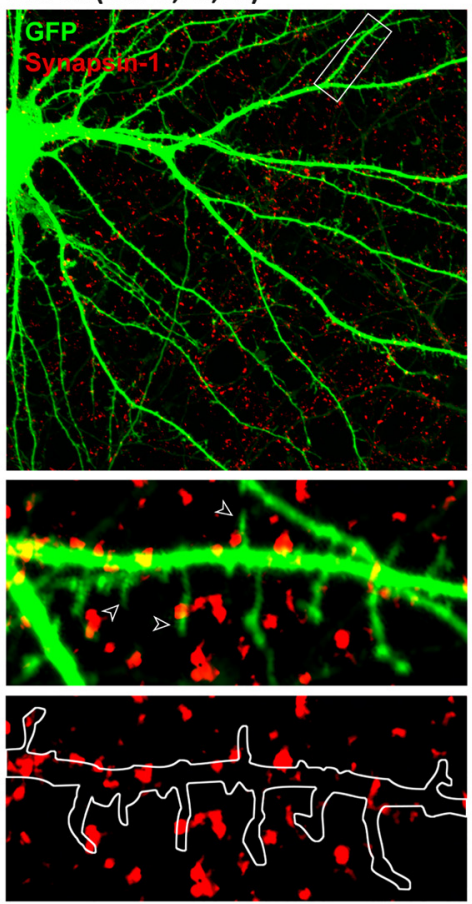

ii.

Synapsin-1

FIGURE 2 | Chronic TTX treatment decreases spine density and Synapsin-1 intensity. GFP-expressing striatal neurons were grown in co-culture at a 1:1 ratio with cortical cells until DIV21, then fixed and stained with anti-GFP (green) and the presynaptic terminal marker Synapsin-1 (red). (A) Experimental timeline for each condition. Cells were treated with $3 \times$ TTX (DIV 7, 14 and 20), $2 \times$ TTX (DIV 7\&14), or received a media removal sham treatment (Control). (B) Top: representative images for each condition (Olympus FV-1000, 60×, $2 \times$ zoom). Middle and bottom: expanded images of the dendritic segment marked by the white rectangle in the corresponding top image (digital zoom). Overlay of GFP fill and Synapsin-1 staining (middle) showing dendritic spines (filled arrowhead), filopodia (open arrowhead), 
FIGURE 2 | Continued

and Synapsin-1-positive presynaptic terminals in red. Outline of GFP filled with Synapsin-1-positive puncta (bottom) to show presynaptic terminals and masked area for quantification. (Ci) There was a significant decrease in dendritic spine density (averaged across three quantified dendritic segments per neuron) in both chronic TTX treatments (Kruskal-Wallis test, ${ }^{* *} p=0.002$; post-hoc $2 \times \Pi \mathrm{X},{ }^{\star} p=0.019,3 \times \Pi \mathrm{X},{ }^{* \star *} p=0.0007$ ) relative to control, but there was no significant difference between the two TTX treatments $(p=$ 0.188). (ii) Filopodia density was not significantly increased following $T X$ treatment relative to control (Kruskal-Wallis test, $p=0.155$ ). (Di,ii) Synapsin-1 cluster size was only significantly reduced in the $2 \times \Pi \mathrm{X}$ condition compared to control (i; Kruskal-Wallis test, ${ }^{\star *} p=0.008$, post-hoc $2 \times \Pi$ TX, ${ }^{* *} p=0.002$, $3 \times \Pi \mathrm{X}, p=0.147)$, and there was no significant difference between treatment groups $(p=0.179)$. Synapsin-1 cluster intensity was reduced in both $\Pi X$ conditions relative to the control (ii; one-way ANOVA,

$F_{(2,115)}=3.409,{ }^{*} p=0.036$; post-hoc, $2 \times \Pi \mathrm{X},{ }^{*} p=0.026$ and $3 \times$ TX, $\left.{ }^{*} p=0.034\right)$, and there was no difference between the two TTX treatment groups $(p=0.791)$

density (Figure 3Dii), or any measures of Synapsin-1 clusters (Figures 3Diii,iv). Thus, a $3 \mathrm{~h}$ silencing period is insufficient to drive structural changes in SPNs, whereas a $24 \mathrm{~h}$ silencing period causes pre- and post-synaptic changes. Interestingly, blocking AMPA and NMDA receptors prevented dendritic protrusion changes, but not Synapsin-1 signal increases. The results suggest that postsynaptic structural plasticity is dependent upon residual, presumably miniature, NMDA/AMPA receptor glutamate signaling over a $24 \mathrm{~h}$ period; in contrast, presynaptic alterations are apparent in response to silencing at terminals on spines (but not filopodia), regardless of NMDA and AMPA receptor signaling.

\section{Chemical LTP Significantly Increases Spine Density, GluA1 Expression, and Alters mEPSC Properties in SPNs}

To determine whether striatal SPNs can exhibit LTP-like changes without the contribution of neuromodulators, we used a pharmacological induction paradigm for NMDARdependent LTP with the NMDAR co-agonist glycine, a protocol similar to what we and others have previously used in cultured hippocampal neurons (Park et al., 2006; Fortin et al., 2010; Brigidi et al., 2014). Cells were treated with glycine for $3 \mathrm{~min}$ in $\mathrm{Mg}^{2+}$-free extracellular solution (cLTP), using a switch to $\mathrm{Mg}^{2+}$-free solution with no glycine addition as a control condition (cLTP control; Figure 4A). There were no significant differences between the cLTP control and the sham control (media removal and replacement; data not shown), and cLTP results were normalized to cLTP control within each culture. Quantification of dendritic protrusions on GFP-expressing SPNs (Figure 4B) revealed that glycine treatment resulted in a significant $\sim 30 \%$ increase in spine density (Figure $4 \mathrm{Ci}$; cLTP ctrl $=1.00 \pm 0.07$ and $\operatorname{cLTP}=1.27 \pm 0.06$ ), with no change in filopodia density (Figure 4Cii). Additionally, cLTP treatment resulted in a significant increase in GluA1 cluster intensity compared to control SPNs (Figure 4Ciii, $1.00 \pm 0.06$ and $1.22 \pm 0.05$ respectively) but no difference in GluA1 cluster density or size (data not shown). Quantification of the percentage of dendritic spines containing clear GluA1 clusters was significantly higher $(70 \%$ increased $)$ in glycine-treated cultures (Figure 4Civ). Presynaptic Synapsin-1 staining did not change following glycine treatment (Figure 4Cv).

Since glycine treatment increased spine density and GluA1 signal, we next assessed functional effects by whole-cell voltage-clamp recordings of miniature excitatory postsynaptic currents (mEPSCs) in control and glycine-treated GFP-filled SPNs (Figure 4Di). Increases in mEPSC frequency (reflective of increased presynaptic glutamate release or active synapses) and increased amplitude (postsynaptic responsiveness) are detected in glycine-treated hippocampal neurons (Brigidi et al., 2014). The frequency of mEPSCs appeared 20\% higher in glycine-treated SPNs, but the trend was not significant (Figure 4Dii), and there were no trends to increased mEPSC amplitude (Figure 4Diii). However, mEPSC event decay time constants (tau) were significantly faster in cLTP SPNs (Figure 4Div); this was not explained by passive membrane properties, which did not differ between the two groups (data not shown). These results suggest that NMDAR activation alone is sufficient to drive LTP-like structural changes in SPNs and to induce significant alterations to the properties of excitatory currents.

\section{DISCUSSION}

The structural responses of SPNs to altered glutamate input were examined within the context of cortico-striatal cocultures, and findings are summarized in the graphical abstract (Figure 5). A reduction in spine density following chronic or medium-term TTX application suggests that excitatory action potential firing in cortico-striatal networks is a crucial regulator of dendritic spines, and thus of excitatory synapse development and/or maintenance on striatal neurons; however, a contributing role of GABA from interneurons or SPNs themselves cannot be ruled out in the present work. NMDARdependent structural LTP-like changes were rapidly induced by glycine stimulation, as evidenced by a $\sim 30 \%$ increase in spine density and GluA1 cluster signals within $30 \mathrm{~min}$. Overall, these pharmacological silencing and plasticity experiments indicate that altering glutamatergic activity is sufficient to drive structural plasticity in SPNs, even in the absence of dopamine and other striatal neuromodulators. Furthermore, while there is an ongoing debate about the role of filopodia as intermediates in spine formation (reviewed in Sala and Segal, 2014), our finding that spine and filopodia densities were not always negatively correlated supports the notion that they are, at least in part, regulated by distinct processes.

We replicate a previous finding that the continuous presence of TTX causes a reversible reduction in spines and increase in filopodia in developing co-cultured SPNs (Segal et al., 2003), and additionally demonstrate that $24 \mathrm{~h}$ silencing is sufficient to drive an increase in filopodia along with a strong trend to decreased spine density. Based on previous reports in corticostriatal co-cultures, SPN spine density increases from $\sim 0.07$ to $0.3-0.4$ spines/ $\mu \mathrm{m}$ between DIV7 and 14 (Burguière et al., 2013; Penrod et al., 2015; Thibault et al., 2016) to $\sim 1$ spine/ $\mu \mathrm{m}$ 

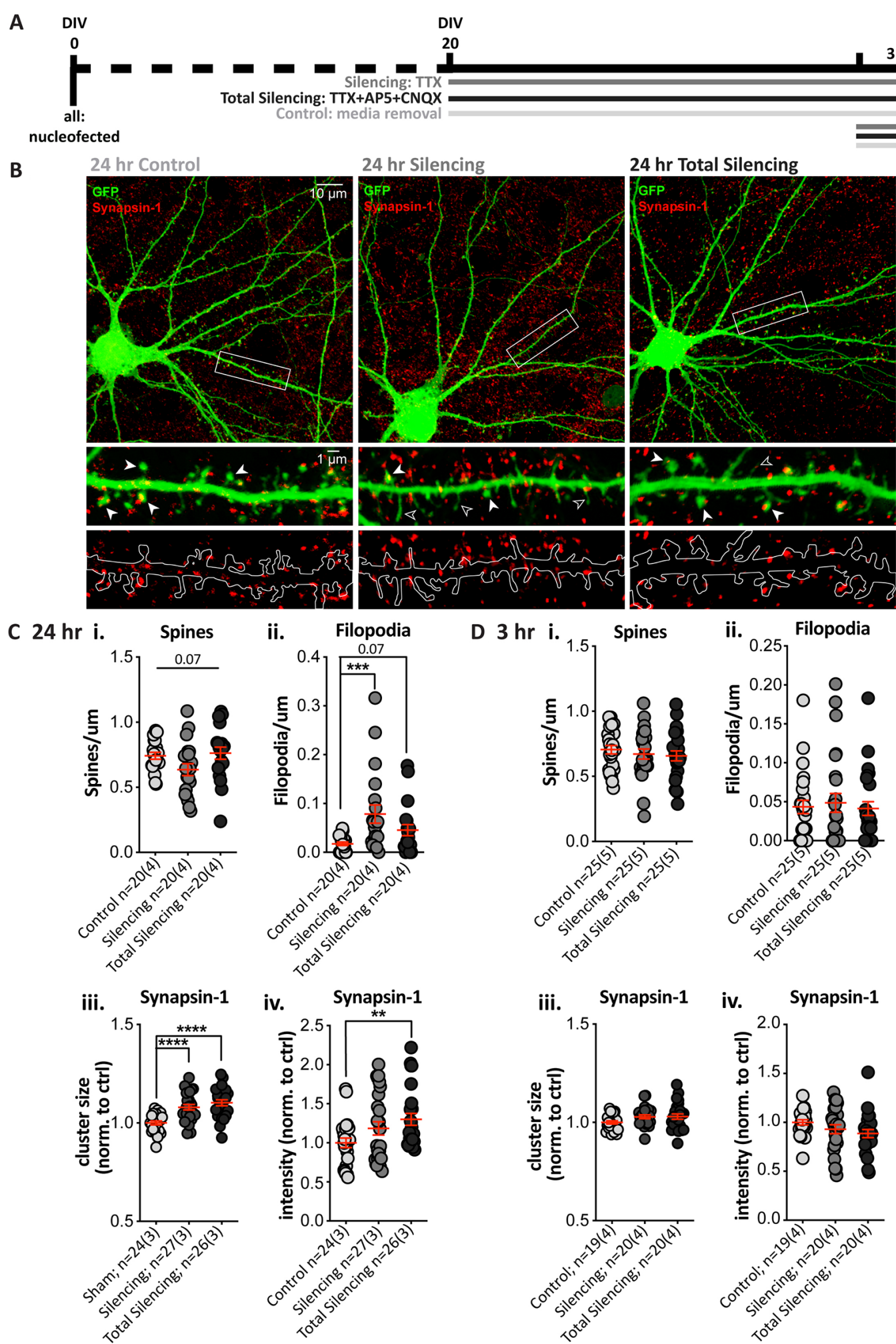

FIGURE 3 | Blocking glutamatergic activity induces structural changes and increases Synapsin-1 cluster intensity after 24, but not 3, hours. (A) Experimental timeline for each condition. TTX (Silencing) or TTX + AP5 + CNQX (Total Silencing) was administered either 24 or $3 \mathrm{~h}$ before fixation on 21 days in vitro (DIV). (B) Top: representative cell images from each condition (Olympus FV-1000, 60×, $2 \times$ zoom). Middle and bottom: expanded images of the dendritic segment outlined in the white rectangle (digital zoom), showing the GFP fill (green) and Synapsin-1-positive presynaptic terminals (red, middle) with visible spines (filled arrowhead) 


\section{FIGURE 3 | Continued}

and filopodia (open arrowhead), and an outline of the GFP fill with Synapsinpuncta (bottom) to illustrate the masked area for quantification. (C) Results from $24 \mathrm{~h}$ blockade. (i) There was no significant difference in spine density, despite a strong trend to a reduction following $\Pi \mathrm{TX}$ treatment (one-way ANOVA, $\left.F_{(2,57)}=2.812,{ }^{\star *} p=0.068\right)$. (ii) There was a significant increase in filopodia density following TTX treatment as compared to control cells (Kruskal-Wallis test, ${ }^{* *} p=0.004$, post-hoc ${ }^{* * *} p=0.0008$ ), which was not observed when AMPA and NMDA receptors were also blocked $(p=0.068)$ and post-hoc comparisons revealed no significant difference between the TXX treatment groups $(p=0.127$ ). (iii,iv) Synapsin- 1 cluster size was significantly increased in both silencing conditions relative to control. (iii) One-way ANOVA, $F_{(2,74)}=15.617,{ }^{* \star * *} p<0.000001$; post-hoc Silencing ${ }^{\star \star \star \star} p<0.0001$ and Total Silencing ${ }^{\star \star \star \star} p<0.000001$ ); and there was no significant difference between treatment groups $(p=0.227)$. The integrated intensity was only significantly increased in the Total Silencing condition compared to control (iv; Kruskal-Wallis test, ${ }^{*} p=0.024$, the post-hoc $p$ value is referred to on the graph ${ }^{* *} p=0.007$ ), whereas the Silencing condition did not differ significantly either from control $(p=0.230)$ or Total Silencing conditions $(p=0.115)$. (Di-iv) No significant changes in dendritic protrusions or Synapsin-1 clusters were observed following a $3 \mathrm{~h}$ treatment. (i) Spines $p$ $=0.6$. (ii) Filopodia $p=0.9$. (iii) Size $p=0.1$. (iv) Intensity $p=0.1$.

by DIV21 (Tian et al., 2010; Penrod et al., 2015). Thus, our reported spine density of $\sim 0.36$ and $\sim 0.46$ spines/ $\mu \mathrm{m}$ at DIV21 following $2 \times$ TTX and $3 \times$ TTX, respectively, suggest a suppression in the maturing spines between DIV7-21; however, it is also possible that newly formed spines are lost or revert to filopodia upon additional TTX applications. In contrast, given the relative stability of spine densities by three weeks in culture (Penrod et al., 2015), our findings following a $24 \mathrm{~h}$ TTX application at DIV20 most likely reflect either a conversion of mature spines to filopodia or distinct regulation of each. Further examination of the mechanisms underlying these changes would benefit from examining SPNs at different developmental stages and at multiple time points following glutamatergic silencing.

That said, the silencing experiments here allow direct comparison of SPN silencing with studies in hippocampal and cortical pyramidal neurons. While a $24 \mathrm{~h}$ silencing period in DIV19 hippocampal neurons did not affect spine density, an increase in spine length was interpreted as a possible conversion to filopodia (Papa and Segal, 1996), in support of the results here. Chronic glutamate blockade has yielded somewhat contradictory results elsewhere; one study reported that TTX application throughout synaptogenesis reduced spine density $\sim 50 \%$ in cultured hippocampal neurons (Kossel et al., 1997), whereas normal synaptogenesis and spine formation was observed in TTX-treated rat hippocampal slice cultures (McKinney et al., 1999; Soares et al., 2013) and in those from transgenic mice entirely lacking presynaptic glutamate release (Sigler et al., 2017). SPNs also respond differently to disrupted glutamatergic transmission in vivo; while hippocampal neurons develop mature spines in the absence of glutamatergic transmission (Sando et al., 2017), reducing glutamatergic release at cortico-striatal synapses in postnatal day (P) eight mice led to a $\sim 40 \%$ reduction in spine density measured at P14-15 (Kozorovitskiy et al., 2012). Such changes may be distinct to spiny GABAergic neurons, as GABAergic cerebellar Purkinje cells also lack spines when cultured in the presence of TTX (Schilling et al., 1991). Future comparison of chronic silencing and glutamate receptor blockade in both SPNs and cortical pyramidal neurons in the same co-culture may prove enlightening. This would enable cell-specific spine/filopodia responses to be assessed under the same treatment paradigms and at the same developmental stages.

Intriguingly, we found increased presynaptic Synapsin-1 cluster size and intensity (but not density) following $24 \mathrm{~h}$ glutamatergic silencing, in contrast to the decrease observed following the chronic blockade. One possibility for this difference is a transient response to $24 \mathrm{~h}$ silencing, related to the immediate pause in the activity-dependent vesicle cycle. Studies of homeostatic plasticity in hippocampal and cortical neurons indicate that $\sim 4-24 \mathrm{~h}$ suppression of action potential firing (or AMPAR activity) increases postsynaptic responses (O’Brien et al., 1998; Turrigiano et al., 1998; Stellwagen and Malenka, 2006; Goel and Lee, 2008; Ibata et al., 2008); however, it remains unclear whether this happens in other neuron types (Rutherford et al., 1997; Kim and Tsien, 2009), as does the extent to which homeostatic presynaptic changes in glutamate release and protein expression occur (Erickson et al., 2006; Wierenga et al., 2006; Turrigiano, 2011; Zhao et al., 2011). Although our results indicate that some presynaptic change occurred at cortico-striatal synapses following silencing, which may precede structural changes (as a trend to increased Synapsin-1 cluster size was visible $3 \mathrm{~h}$ post-treatment), the interpretation is limited by our choice of presynaptic marker. Given that Synapsin-1 is present at both glutamatergic and GABAergic synapses, we cannot distinguish whether our silencing paradigms differentially altered excitatory or inhibitory input onto SPNs; moreover, opposite regulation of these inputs could cancel one another, or mask additional effects in our readouts. To verify whether the presynaptic input changes observed here were occurring at excitatory synapses, we conducted additional analysis in which we quantified Synapsin-1 puncta on dendritic spines and filopodia only. While this strongly suggests that the observed Synapsin-1 changes were indicative of plasticity at excitatory synapses, future experiments would benefit from staining for synapse-specific pre- and post-synaptic markers to distinguish between inhibitory and excitatory synapses (for example VGLUT1 and PSD95 vs. VGAT and gephyrin, respectively; Rao and Craig, 1997; Levinson and El-Husseini, 2005). This, in combination with electrophysiological recordings of both excitatory and inhibitory post-synaptic currents, would provide more insight as to how different forms of glutamatergic blockade (chronic vs. acute; burst firing vs. receptor blockade) affect both structural and functional plasticity at synapses on SPNs. Future experiments could additionally examine activity-dependant effects on AMPA and NMDA receptor subunit composition and subcellular distribution; given that alterations to these are hallmarks of activity blockade in other glutamatergic neurons (Rao and Craig, 1997; Ehlers, 2003; Soares et al., 2013), it is worth investigating whether similar changes occur in SPNs. Nonetheless, examining 
A

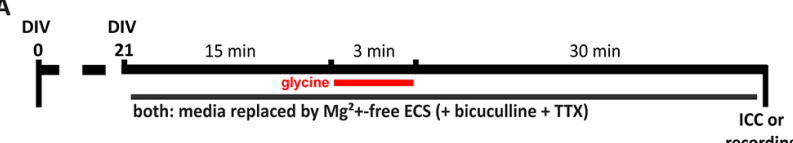

B
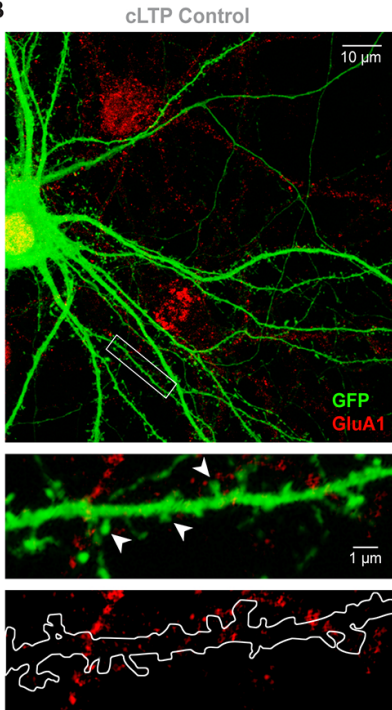

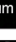

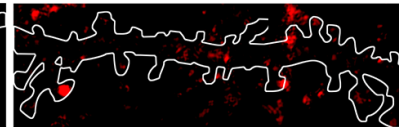

D i.
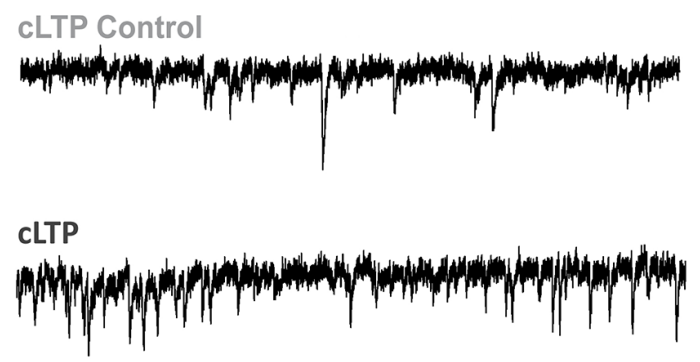

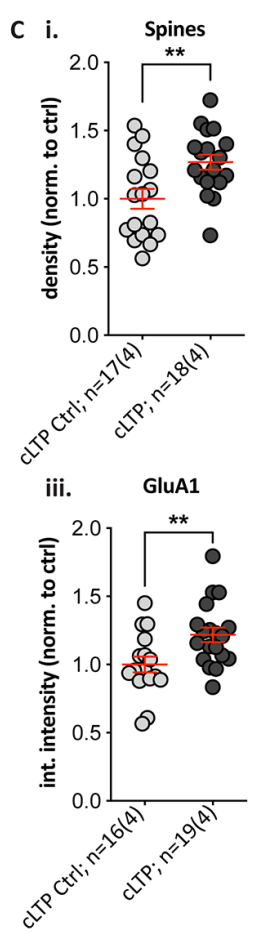

ii.

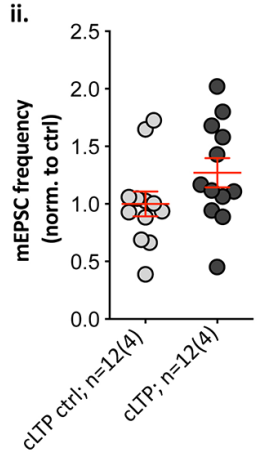

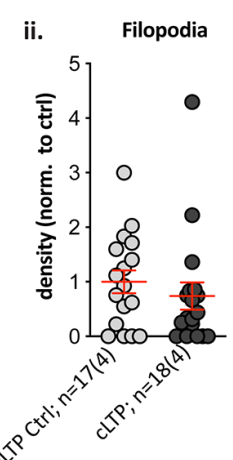
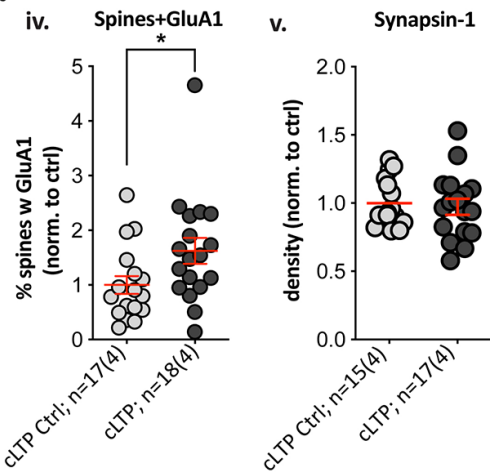

iii.

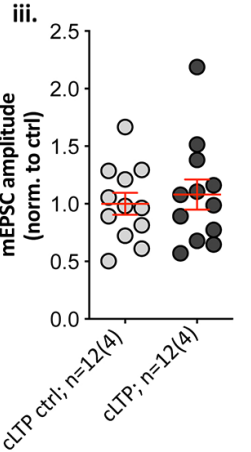

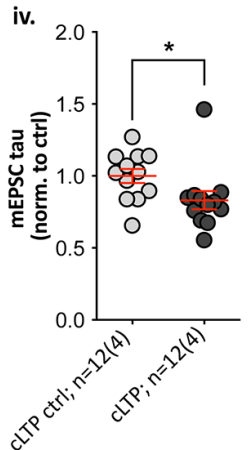

FIGURE 4 | A 3-min glycine CLTP induction protocol induced spine density and GluA1 cluster increases within 30 min and altered the decay time of miniature events. (A) Experimental timeline. Following glycine or control treatment, cells were fixed and immunostained with anti-GFP (green) and the presynaptic terminal marker Synapsin-1 (not shown) or the postsynaptic AMPA receptor subunit GluA1 (red). Separate coverslips were used for whole-cell voltage-clamp recordings. (B) Top: representative images (Olympus FV-1000, 60×, 2× zoom) of control (cLTP ctrl) and +glycine (cLTP) cells. Middle and bottom: expanded images (digital zoom) depicting the dendritic segment outlined in the white rectangle above. Middle row images show GFP fill with visible spines and GluA1 clusters, and in the lower panel, the GFP fill is outlined to depict the masked area for puncta quantification. (C) Structural and synaptic marker changes following glycine treatment in Mg ${ }^{2+}$-free ECS. (i,ii) The analysis revealed a $\sim 30 \%$ increase in spine density relative to control-treated SPNs. (i) Unpaired $t$-test, ${ }^{* \star} p=0.006$; whereas no change was observed in filopodia density. (ii) $p=0.2$. (iii,iv) GluA1 cluster intensity was significantly increased in glycine-treated relative to control SPNs. (iii) Unpaired $t$-test, ${ }^{* \star} p=0.009$; as was the percentage of spines colocalized with GluA1 clusters in glycine treated SPNs. (iv) Mann-Whitney test, ${ }^{*} p=0.022$. (v) No changes in Synapsin-1 cluster density were observed. (D) Whole-cell patch-clamp recordings from cLTP and control SPNs. (i) Representative traces showing miniature excitatory postsynaptic currents (mEPSCs) in the control (top) and glycine-treated (bottom) SPN. (ii,iii) Despite a trend, there was no significant difference in mEPSC frequency. (ii) $p=0.1$ and no change in amplitudes. (iii) $p=0.5$. (iv) The mEPSC decay time (tau) was significantly faster, following glycine treatment (Mann-Whitney test, ${ }^{\star} p=0.015$ ).

the overall change in presynaptic input onto SPNs, in parallel to quantifying dendritic protrusions, highlighted an interesting difference between the response to chronic and $24 \mathrm{~h}$ glutamatergic blockade.

Our observations following the additional blockade of AMPARs and NMDARs during $24 \mathrm{~h}$ silencing suggest a potential disconnect between the spine and filopodia dynamics. While blocking ionotropic glutamate receptors prevented any suggestion of a change in spine density, a very strong trend to increased filopodia density remained. This is in agreement with results in cortical pyramidal and hippocampal neurons, where NMDAR activity is required for activity-dependent spinogenesis (Fischer et al., 2000; Kwon and Sabatini, 2011) and spine shrinkage/loss (Nägerl et al., 2004; Zhou et al., 2004; Oh et al., 2013), and where AMPAR activity regulates spine motility (Fischer et al., 2000) and maintenance (McKinney et al., 1999). 

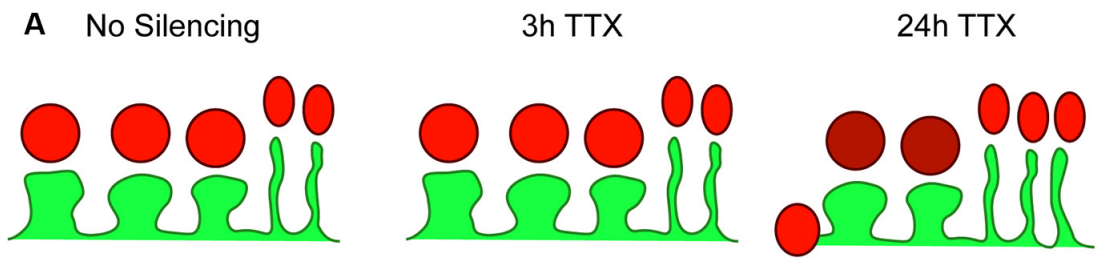

\section{2 weeks TTX}

3h TTX+AP5+CNQX

24h TTX+AP5+CNQX

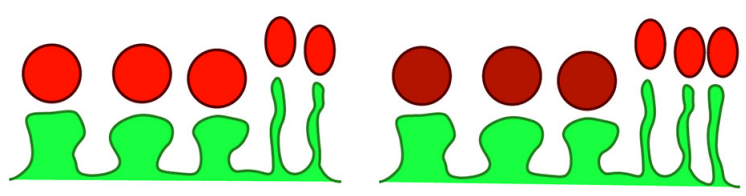

B

cLTP Control

cLTP 3min Glycine
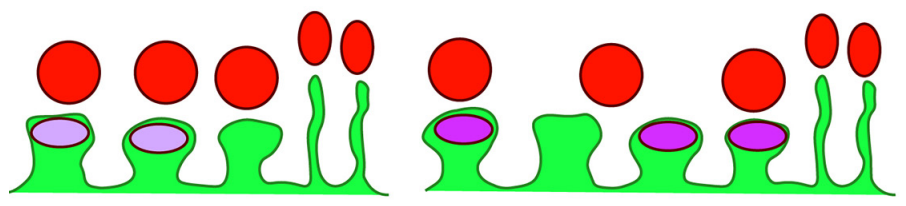

FIGURE 5 | Summary of results. (A) Cartoon depicting basal (no silencing) striatal projection neuron dendrite (SPN; green) with three postsynaptic spines and two filopodia, with presynaptic Synapsin-1 signal shown in red. There were no changes detected following $3 \mathrm{~h} T \mathrm{TX}$ silencing or $3 \mathrm{~h} T \mathrm{TX}+\mathrm{AP} 5+\mathrm{CNQX}$. Silencing with TTX for $24 \mathrm{~h}$ increased Synapsin-1 signal intensity specifically on dendritic spines (darker red), and began the process of spine elimination, without changes to filopodia density. The additional block of spontaneous glutamate receptor activation (AP5 + CNQX) prevented reductions in spine density but not increased Synapsin-1 signal intensity. Chronic silencing with 2-week exposure to TTX dramatically reduced spine density and decreased Synapsin-1 signal intensity; specifically, that associated with dendritic spines and dendrites. (B) Cartoon of control (no glycine) SPN with three spines and two filopodia with postsynaptic GluA1 AMPA receptor signal shown in purple. A chemical LTP protocol (3 min glycine) increased spine density, GluA1 signal intensity, and the percentage of spines with clearly detected GluA1 clusters, without changing presynaptic cluster density.

Distinct effects on spine and filopodia dynamics have also been observed elsewhere; blocking AMPA receptors reduced spine density in hippocampal slices 7 days post-treatment, whereas NMDAR blockade had no effect on spines, but instead caused the appearance of filopodia-like protrusions (McKinney et al., 1999).

Beyond methodological variability, different responses to glutamate receptor blockade may arise from the existence of different filopodia sub-types (Portera-Cailliau et al., 2003; Richards et al., 2005), as well as the effects of developmental stage on filopodia dynamics (Sala and Segal, 2014); these distinctions could be tested in future experiments. It is also worth considering that some differences between blocking burst firing vs. all glutamatergic activity may arise from extrasynaptic receptors; while we assume antagonists are most effective in blocking spontaneous glutamate release and activation of receptors close to release sites, we cannot rule out the possibility that silencing extrasynaptic receptor activation through ambient glutamate in the media may contribute. Regardless, our results extend the literature on the effects of glutamatergic receptor blockade to striatal SPNs, demonstrating that, as at other glutamatergic synapses, spine pruning is an active process requiring ongoing low-level glutamate activity, and can be uncoupled from filopodia formation.

The role of NMDARs in striatal activity-dependent plasticity has been revealed primarily by slice electrophysiology (reviewed in Perrin and Venance, 2019). Although LTD was initially considered the dominant form of plasticity at cortico-striatal synapses, many reports have since shown that high-frequency stimulation can result in either NMDAR-dependent LTP, or mGluR-dependent LTD (Calabresi et al., 1996; Spencer and Murphy, 2000; Tang et al., 2001; Reynolds and Wickens, 2002; Wang et al., 2006; Sergeeva et al., 2007; Li et al., 2009; Lovinger and Mathur, 2012; Johnson et al., 2017). However, debate remains as to whether dopamine or other neuromodulators are necessary for the expression of LTP (Spencer and Murphy, 2000; Calabresi et al., 2007; Li et al., 2009; Lovinger, 2010; Burguière et al., 2013; Park et al., 2014; Cerovic et al., 2015). Only a few studies have specifically examined activity-dependent spine alterations in the context of AMPAR trafficking in SPNs (Kozorovitskiy et al., 2012, 2015; Matikainen-Ankney et al., 2018), and, to our knowledge, only one other study has done so in the absence of dopamine (Burguière et al., 2013). Here, we show that LTP-like changes in SPNs can be driven by 
NMDAR activity alone, as the NMDAR co-agonist glycine (in the absence of $\mathrm{Mg}^{2+}$ ) produced a rapid increase in dendritic spines and associated GluA1 expression. Thus, even in the absence of dopamine, SPNs show a similar response to NMDAR stimulation compared to principle excitatory neurons, in which LTP induction by glutamate uncaging (Matsuzaki et al., 2004; Yang et al., 2008) or chemical paradigms (Lin et al., 2004; Huang et al., 2005; Park et al., 2006; Sharma et al., 2006; Korkotian and Segal, 2007; Fortin et al., 2012; Brigidi et al., 2014) leads to increased spine head volume or de novo spine formation without filopodial intermediates (Kwon and Sabatini, 2011). However, a contributing role of non-neurotransmitter neuromodulators within the culture media, such as BDNF, cannot be discounted in the present findings.

The observed increase to GluA1 cluster intensity and association within spines also suggests a functional change, consistent with results in hippocampal neurons showing trafficking and membrane insertion of GluA1-containing AMPARs in spines following LTP induction (Shi et al., 2001; Malinow and Malenka, 2002; Matsuo et al., 2008; Fortin et al., 2010). However, electrophysiological measures of activitydependent changes here were less clear. Whole-cell patch-clamp recordings $\sim 30-60 \mathrm{~min}$ following treatment revealed variable effects on mEPSC frequency, although a trend to increased frequency in glycine-treated SPNs was observed. Moreover, we found no indication of increased mEPSC amplitude following glycine, despite the increased GluA1 signal. A potential reason for this is that the synaptic effects of glycine stimulation are not fully captured by measuring quantal (miniature) glutamatergic transmission, and that changes in evoked activity would be more apparent, given the growing body of literature suggesting that these are mechanistically distinct (Ramirez and Kavalali, 2011; Kavalali, 2015; Abrahamsson et al., 2017; Andreae and Burrone, 2018; Chanaday and Kavalali, 2018). Alternatively, the observed increase in spine density could precede functional changes requiring associated new presynaptic elements (as Synapsin-1 density did not increase); apropos, spine enlargement before AMPAR insertion has been observed following chemical LTP induction in hippocampal slices (Kopec et al., 2006).

In a separate study in which we used the same chemical LTP protocol on cultured hippocampal neurons, we found increased mEPSC amplitude and frequency, which correlated with increased spine width and density 30-60 min after glycine stimulation (Brigidi et al., 2014). It is thus possible that SPNs, unlike hippocampal neurons, require neuromodulators to fully express synaptic LTP in terms of current flux, while structural plasticity can be induced by NMDAR activation alone. In support of this, glutamate uncaging alone leads to spinogenesis in SPNs $\sim 50 \%$ of the time, whereas D1 or A2a receptor agonists significantly increase the probability of novel spines and functional synapses (as evidenced by increased mEPSC frequency) in D1R and D2R SPNs, respectively (Kozorovitskiy et al., 2015). Nevertheless, the significant decrease in mEPSC event-decay constant following glycine treatment indicates that some functional change occurred at corticostriatal synapses, possibly reflecting altered glutamate receptor subunit composition or phosphorylation (Lambolez et al., 1996;
Banke et al., 2000; Chater and Goda, 2014). GluA2-lacking, calcium-permeable AMPARs exhibit faster decay kinetics than those containing GluA2 (reviewed in Diering and Huganir, 2018), and multiple studies have reported their integration at specific synapses, including cortico-striatal ones, during LTP induction (Lamsa et al., 2007; Soares et al., 2013; Ma et al., 2018; Park et al., 2018; Benke and Traynelis, 2019). Thus, the faster decay of mEPSCs in glycine-treated co-cultures, together with the increased GluA1 signal in spines, may reflect the activitydependent insertion of calcium-permeable AMPARs in SPNs. Future work could extend these findings by recording evoked vs. miniature EPSCs, verifying differences in SPN subtype, and/or additionally examining the response to D1 and A2a receptor agonists. Regardless, our results show that NMDAR activation drives rapid structural, and some electrophysiological changes at cortico-striatal synapses.

This study presents an examination of activity-dependent structural development and plasticity within GABAergic striatal projection neurons. Chronic and short-term glutamatergic manipulations to co-cultured SPNs provides a comparison with similar studies in hippocampal and cortical pyramidal neurons and highlights the distinct but overlapping regulation of spine and filopodial activity-dependent plasticity. In particular, we show that SPN structural plasticity occurs within $24 \mathrm{~h}$ of glutamate activity blockade, and within $30 \mathrm{~min}$ of a 3-min NMDAR activation by glycine, even in the absence of dopamine; thus, the cortico-striatal co-culture system is useful for examining the specific role of glutamate receptor activity in shaping SPN physiology and cortico-striatal synapses. While our primary aim was to provide a characterization of structural plasticity in SPNs and how these may differ from principal excitatory neurons, we offer several ideas on how these assays can be refined and built upon. These could easily be applied to examining activitydependent plasticity in disease models, particularly those in which altered glutamatergic transmission and aberrant structural plasticity may play a pathophysiological role, and in which SPNs have shown distinct vulnerability.

\section{DATA AVAILABILITY STATEMENT}

The raw data supporting the conclusions of this article will be made available by the authors upon request, without undue reservation.

\section{ETHICS STATEMENT}

The animal study was reviewed and approved by Canadian Council on Animal Care.

\section{AUTHOR CONTRIBUTIONS}

NK and AM designed the study and co-wrote the manuscript. With supervision from AM, NK conducted all experiments, analyzed data, interpreted results, and made figures. MW and LQ-R helped with image processing and analysis. MF provided scientific input and supervisory support throughout. All authors contributed to the article and approved the submitted version. 


\section{FUNDING}

This work was funded by the Natural Sciences and Engineering Research Council of Canada (Canadian Graduate Student scholarship for NK), Parkinson's Society Canada (scholarship for NK; new investigator grant for AM), the Canadian Foundation for Innovation (experimental equipment), the Fonds de la recherche en santé du Quebec (salary award AM), McGill University (AM) and the University of British Columbia (NK, AM, MF).

\section{ACKNOWLEDGMENTS}

We would like to thank Liping Cao and Brittney Smaila of the Centre for Applied Neurogenetics for their help with cell culturing and genotyping, and Lilly Zhang and Lynn Raymond for the use of D2 BAC cultures.

\section{SUPPLEMENTARY MATERIAL}

The Supplementary Material for this article can be found online at: https://www.frontiersin.org/articles/10.3389/fncel.2021.5690 31/full\#supplementary-material.

\section{REFERENCES}

Abrahamsson, T., Chou, C. Y. C., Li, S. Y., Mancino, A., Costa, R. P., Brock, J. A., et al. (2017). Differential regulation of evoked and spontaneous release by presynaptic NMDA receptors. Neuron 96, 839-855. doi: 10.1016/j.neuron.2017. 09.030

Ade, K. K., Wan, Y., Chen, M., Gloss, B., and Calakos, N. (2011). An improved BAC transgenic fluorescent reporter line for sensitive and specific identification of striatonigral medium spiny neurons. Front. Syst. Neurosci. 5, 1-9. doi: 10.3389/fnsys.2011.00032

Andreae, L. C., and Burrone, J. (2014). The role of neuronal activity and transmitter release on synapse formation. Curr. Opin. Neurobiol. 27, 47-52. doi: 10.1016/j.conb.2014.02.008

Andreae, L. C., and Burrone, J. (2018). The role of spontaneous neurotransmission in synapse and circuit development. J. Neurosci. Res. 96, 354-359. doi: 10.1002/ jnr. 24154

Arstikaitis, P., Gauthier-Campbell, C., Huang, K., El-Husseini, A., and Murphy, T. H. (2011). Proteins that promote filopodia stability, but not number, lead to more axonal-dendritic contacts. PLoS One 6:e16998. doi: 10.1371/journal.pone.0016998

Banke, T. G., Bowie, D., Lee, H. K., Huganir, R. L., Schousboe, A., and Traynelis, S. F. (2000). Control of GluR1 AMPA receptor function by cAMP-dependent protein kinase. J. Neurosci. 20, 89-102. doi: 10.1523/JNEUROSCI.20-01-00089.2000

Beccano-Kelly, D. A., Kuhlmann, N., Tatarnikov, I., Volta, M., Munsie, L. N., Chou, P., et al. (2014). Synaptic function is modulated by LRRK2 and glutamate release is increased in cortical neurons of G2019S LRRK2 knock-in mice. Front. Cell. Neurosci. 8:301. doi: 10.3389/fncel.2014.00301

Benke, T., and Traynelis, S. F. (2019). AMPA-type glutamate receptor conductance changes and plasticity: still a lot of noise. Neurochem. Res. 44, 539-548. doi: 10.1007/s11064-018-2491-1

Blackwell, K. T., Salinas, A. G., Tewatia, P., English, B., Hellgren Kotaleski, J., and Lovinger, D. M. (2019). Molecular mechanisms underlying striatal synaptic plasticity: relevance to chronic alcohol consumption and seeking. Eur. J. Neurosci. 49, 768-783. doi: 10.1111/ejn.13919
SUPPLEMENTARY FIGURE 1 | Synapsin-1 total dendritic density, and spine-/filopodia-specific changes in Synapsin-1 puncta following chronic and acute silencing. Additional analysis to quantify the effects of glutamatergic silencing on Synapsin-1 puncta at select dendritic protrusions as opposed to the full dendritic arbor. In a subset of images (5-10 per culture) in chronic (A) and acute (B) silencing experiments, ROls were created around a sample of spines and any visible filopodia on secondary or tertiary dendrites of GFP-expressing SPNs, to calculate the integrated density (intensity) of Synapsin-1 in the corresponding raw images. (A) There were no changes in total dendritic Synapsin-1 cluster density in chronic TTX silencing experiments; analysis of dendritic spines and filopodia showed a significant reduction in Synapsin-1 integrated intensity on spines following silencing (Kruskal-Wallis test,

$\left.{ }_{* * * *} p<0.0001\right)$, with post-hoc tests indicating that the $3 \times$ TTX condition was reduced compared to the $2 \times$ TTX condition and control (Uncorrected Dunn's test; ${ }^{* *} p=0.008$ and ${ }^{* * * *} p<0.0001$, respectively), whereas there was no significant difference between $2 \times T$ TX and control $(p=0.291)$. In contrast, Synapsin-1 signal on filopodia was significantly higher in the $2 \times T$ X condition when compared to control (Kruskal-Wallis test, ${ }^{* * *} p=0.0008$; post-hoc Uncorrected Dunn's test, $\left.{ }^{*} p=0.034\right)$ and compared to the $3 \times T$ X condition $\left({ }^{* * *} p=0.0002\right)$. (B) Acute (24 h) silencing experiments, with separate analysis for dendritic spines and filopodia. There were no changes in total dendritic Synapsin-1 cluster density in $24 \mathrm{~h}$ acute TTX silencing experiments. On spines, both the Silencing (TTX only) and Total Silencing (TTX + APV + CNQX) conditions showed significantly increased Synapsin-1 integrated intensity compared to control (Kruskal-Wallis test, ${ }^{* * * *} p<0.0001$; post-hoc Uncorrected Dunn's Test, ${ }^{* * * *} p<0.0001$ for both). Additionally, the Total Silencing condition had a significantly greater increase compared to the Silencing condition (*** $p=0.0003$ ). In contrast, there was no significant difference in Synapsin-1 signal on filopodia (Kruskal-Wallis test, $p=0.838)$.

Bliss, T. V. P., and Collingridge, G. L. (1993). A synaptic model of memory: long-term potentiation in the hippocampus. Nature 361, 31-39. doi: $10.1038 / 361031 \mathrm{a} 0$

Bourne, J. N., and Harris, K. M. (2008). Balancing structure and function at hippocampal dendritic spines. Ann. Rev. Neurosci. 31, 47-67. doi: 10.1146/annurev.neuro.31.060407.125646

Brigidi, G. S., Sun, Y., Beccano-Kelly, D., Pitman, K., Mobasser, M., Borgland, S. L., et al. (2014). Palmitoylation of $\delta$-catenin by DHHC5 mediates activity-induced synapse plasticity. Nat. Neurosci. 17, 522-532. doi: 10.1038/nn.3657

Burguière, A., De Bundel, D., Valjent, E., Roger, J., Smolders, I., Fagni, L., et al. (2013). Combination of group I mGlu receptors antagonist with dopaminergic agonists strengthens the synaptic transmission at corticostriatal synapses in culture. Neuropharmacology 66, 151-157. doi: 10.1016/j.neuropharm.2012. 03.017

Calabresi, P., Picconi, B., Tozzi, A., and Di Filippo, M. (2007). Dopamine-mediated regulation of corticostriatal synaptic plasticity. Trends Neurosci. 30, 211-219. doi: 10.1016/j.tins.2007.03.001

Calabresi, P., Pisani, A., Centonze, D., and Bernardi, G. (1997). Synaptic plasticity and physiological interactions between dopamine and glutamate in the striatum. Neurosci. Biobehav. Rev. 21, 519-523. doi: 10.1016/s01497634(96)00029-2

Calabresi, P., Pisani, A., Mercuri, N. B., and Bernardi, G. (1996). The corticostriatal projection: from synaptic plasticity to dysfunctions of the basal ganglia. Trends Neurosci. 19, 19-24. doi: 10.1016/0166-2236(96)81862-5

Cerovic, M., Bagetta, V., Pendolino, V., Ghiglieri, V., Fasano, S., Morella, I., et al. (2015). Derangement of ras-guanine nucleotide-releasing factor 1 (Ras-GRF1) and extracellular signal-regulated kinase (ERK) dependent striatal plasticity in L-DOPA-induced dyskinesia. Biol. Psychiatry 77, 106-115. doi: 10.1016/j. biopsych.2014.04.002

Chanaday, N. L., and Kavalali, E. T. (2018). Optical detection of three modes of endocytosis at hippocampal synapses. eLife 7, 1-24. doi: 10.7554/eLife. 36097

Chater, T. E., and Goda, Y. (2014). The role of AMPA receptors in postsynaptic mechanisms of synaptic plasticity. Front. Cell. Neurosci. 8, 1-14. doi: 10.3389/fncel.2014.00401 
Choi, B. J., Imlach, W. L., Jiao, W., Wolfram, V., Wu, Y., Grbic, M., et al. (2014). Miniature neurotransmission regulates Drosophila synaptic structural maturation. Neuron 82, 618-634. doi: 10.1016/j.neuron.2014.03.012

Choi, S. H., Kim, Y. H., Hebisch, M., Sliwinski, C., Lee, S., D’Avanzo, C., et al. (2014). A three-dimensional human neural cell culture model of Alzheimer's disease. Nature 515, 274-278. doi: 10.1038/nature13800

Day, M., Wang, Z., Ding, J., An, X., Ingham, C. A., Shering, A. F., et al. (2006). Selective elimination of glutamatergic synapses on striatopallidal neurons in Parkinson disease models. Nat. Neurosci. 9, 251-259. doi: 10.1038/ nn 1632

Diering, G. H., and Huganir, R. L. (2018). The AMPA receptor code of synaptic plasticity. Neuron 100, 314-329. doi: 10.1016/j.neuron.2018.10.018

Doig, N. M., Moss, J., and Bolam, J. P. (2010). Cortical and thalamic innervation of direct and indirect pathway medium-sized spiny neurons in mouse striatum. J. Neurosci. 30, 14610-14618. doi: 10.1523/JNEUROSCI.1623-10.2010

Ehlers, M. D. (2003). Activity level controls postsynaptic composition and signaling via the ubiquitin-proteasome system. Nat. Neurosci. 6, 231-242. doi: $10.1038 / \mathrm{nn} 1013$

Engert, F., and Bonhoeffer, T. (1999). Dendritic spine changes associated with hippocampal long-term synaptic plasticity. Nature 399, 66-70. doi: 10.1038/19978

Erickson, J. D., De Gois, S., Varoqui, H., Schafer, M. K. H., and Weihe, E. (2006). Activity-dependent regulation of vesicular glutamate and GABA transporters: a means to scale quantal size. Neurochem. Int. 48, 643-649. doi: 10.1016/j.neuint. 2005.12.029

Fasano, C., Bourque, M.-J., Lapointe, G., Leo, D., Thibault, D., Haber, M., et al. (2013). Dopamine facilitates dendritic spine formation by cultured striatal medium spiny neurons through both D1 and D2 dopamine receptors. Neuropharmacology 67, 432-443. doi: 10.1016/j.neuropharm.2012.11.030

Fischer, M., Kaech, S., Wagner, U., Brinkhaus, H., and Matus, A. (2000). Glutamate receptors regulate actin-based plasticity in dendritic spines. Nat. Neurosci. 3, 887-894. doi: 10.1038/78791

Fishbein, I., and Segal, M. (2011). Active cortical innervation protects striatal neurons from slow degeneration in culture. J. Neural Transm. 118, 445-451. doi: 10.1007/s00702-010-0505-5

Fortin, D. A., Davare, M. A., Srivastava, T., Brady, J. D., Nygaard, S., Derkach, V. A., et al. (2010). Long-term potentiation-dependent spine enlargement requires synaptic $\mathrm{Ca}^{2+}$-permeable AMPA receptors recruited by CaM-kinase I. J. Neurosci. 30, 11565-11575. doi: 10.1523/JNEUROSCI.174610.2010

Fortin, D. A., Srivastava, T., and Soderling, T. R. (2012). Structural modulation of dendritic spines during synaptic plasticity. Neuroscientist 18, 326-341. doi: 10.1177/1073858411407206

Friend, D. M., and Kravitz, A. V. (2014). Working together: basal ganglia pathways in action selection. Trends Neurosci. 37, 301-303. doi: 10.1016/j.tins.2014. 04.004

Gerfen, C. R., and Surmeier, D. J. (2011). Modulation of striatal projection systems by dopamine. Ann. Rev. Neurosci. 34, 441-466. doi: 10.1146/annurev-neuro061010-113641

Goel, A., and Lee, H.-K. (2008). Persistence of experience-induced homeostatic synaptic plasticity through adulthood in superficial layers of mouse visual cortex. J. Neurosci. 27, 6692-6700. doi: 10.1523/JNEUROSCI.503806.2007

Goldin, M., Segal, M., and Avignone, E. (2001). Functional plasticity triggers formation and pruning of dendritic spines in cultured hippocampal networks. J. Neurosci. 21, 186-193. doi: 10.1523/JNEUROSCI.21-0100186.2001

Graybiel, A. M., Canales, J. J., and Capper-Loup, C. (2000). Levodopa-induced dyskinesias and dopamine-dependent stereotypies: a new hypothesis. Trends Neurosci. 23, S71-S77. doi: 10.1016/s1471-1931(00)00027-6

Hartman, K. N., Pal, S. K., Burrone, J., and Murthy, V. N. (2006). Activitydependent regulation of inhibitory synaptic transmission in hippocampal neurons. Nat. Neurosci. 9, 642-649. doi: 10.1038/nn1677

Huang, Y., Man, H. Y., Sekine-Aizawa, Y., Han, Y., Juluri, K., Luo, H., et al. (2005). S-nitrosylation of N-ethylmaleimide sensitive factor mediates surface expression of AMPA receptors. Neuron 46, 533-540. doi: 10.1016/j.neuron. 2005.03 .028
Huberman, A. D., Feller, M. B., and Chapman, B. (2008). Mechanisms underlying development of visual maps and receptive fields. Annu. Rev. Neurosci. 31, 479-509. doi: 10.1146/annurev.neuro.31.060407.125533

Ibata, K., Sun, Q., and Turrigiano, G. G. (2008). Rapid synaptic scaling induced by changes in postsynaptic firing. Neuron 57, 819-826. doi: 10.1016/j.neuron. 2008.02.031

Johnson, K. A., Mateo, Y., and Lovinger, D. M. (2017). Metabotropic glutamate receptor 2 inhibits thalamically-driven glutamate and dopamine release in the dorsal striatum. Neuropharmacology 117, 114-123. doi: 10.1016/j.neuropharm. 2017.01.038

Katz, L. C., and Shatz, C. J. (1996). Synaptic activity and the construction of circuits. Science 274, 1133-1138. doi: 10.1126/science.274.5290.1133

Kaufman, A. M., Milnerwood, A. J., Sepers, M. D., Coquinco, A., She, K., Wang, L., et al. (2012). Opposing roles of synaptic and extrasynaptic NMDA receptor signaling in cocultured striatal and cortical neurons. J. Neurosci. 32, 3992-4003. doi: 10.1523/JNEUROSCI.4129-11.2012

Kavalali, E. T. (2015). The mechanisms and functions of spontaneous neurotransmitter release. Nat. Rev. Neurosci. 16, 5-16. doi: 10.1038/nrn3875

Kawaguchi, Y., and Kubota, Y. (1997). GABAergic cell subtypes and their synaptic connections in rat frontal cortex. Cereb. Cortex 7, 476-486. doi: $10.1093 /$ cercor/7.6.476

Kim, J., and Tsien, R. (2009). Synapse-specific adaptations to inactivity in hippocampal circuits achieve homeostatic gain control while dampening network reverberation. Neuron 58, 925-937. doi: 10.1016/j.neuron.2008.05.009

Kincaid, A. E., Zheng, T., and Wilson, C. J. (1998). Connectivity and convergence of single corticostriatal axons. J. Neurosci. 18, 4722-4731. doi: 10.1523/JNEUROSCI.18-12-04722.1998

Kopec, C. D., Li, B., Wei, W., Boehm, J., and Malinow, R. (2006). Glutamate receptor exocytosis and spine enlargement during chemically induced long-term potentiation. J. Neurosci. 26, 2000-2009. doi: 10.1523/JNEUROSCI. 3918-05.2006

Korkotian, E., and Segal, M. (2007). Morphological constraints on calcium dependent glutamate receptor trafficking into individual dendritic spine. Cell Calcium 42, 41-57. doi: 10.1016/j.ceca.2006.11.006

Kossel, A. H., Williams, C. V., Schweizer, M., and Kater, S. B. (1997). Afferent innervation influences the development of dendritic branches and spines via both activity-dependent and non-activity-dependent mechanisms. J. Neurosci. 17, 6314-6324. doi: 10.1523/JNEUROSCI.17-16-06314.1997

Kozorovitskiy, Y., Peixoto, R., Wang, W., Saunders, A., and Sabatini, B. L. (2015). Neuromodulation of excitatory synaptogenesis in striatal development. eLife 4:e10111. doi: 10.7554/eLife.10111

Kozorovitskiy, Y., Saunders, A., Johnson, C. A., Lowell, B. B., and Sabatini, B. L. (2012). Recurrent network activity drives striatal synaptogenesis. Nature 485 , 646-650. doi: 10.1038/nature11052

Kreitzer, A. C. (2009). Physiology and pharmacology of striatal neurons. Ann. Rev. Neurosci. 32, 127-147. doi: 10.1146/annurev.neuro.051508.135422

Kreitzer, A. C., and Malenka, R. C. (2007). Endocannabinoid-mediated rescue of striatal LTD and motor deficits in Parkinson's disease models. Nature 445, 643-647. doi: 10.1038/nature05506

Kwon, H.-B., and Sabatini, B. (2011). Glutamate induces de novo growth of functinal spines in developing cortex. Nature 33, 100-104. doi: 10.1038/nature09986

Lalchandani, R. R., van der Goes, M.-S., Partridge, J. G., and Vicini, S. (2013). Dopamine D2 receptors regulate collateral inhibition between striatal medium spiny neurons. J. Neurosci. 33, 14075-14086. doi: 10.1523/JNEUROSCI.069213.2013

Lambolez, B., Ropert, N., Ferrais, D., Rossier, J., and Hestrin, S. (1996). Correlation between kinetics and RNA splicing of $\alpha$-amino-3-hydroxy-5-methylisoxazole4-propionic acid receptors in neocortical neurons. Proc. Natl. Acad. Sci. U S A 93, 1797-1802. doi: 10.1073/pnas.93.5.1797

Lamsa, K. P., Heeroma, J. H., Somogyi, P., Rusakov, D. A., and Kullmann, D. M. (2007). Anti-hebbian long-term potentiation in the hippocampal feedback inhibitory circuit. Science 315, 1262-1266. doi: 10.1126/science. 1137450

LeVay, S., Wiesel, T. N., and Hubel, D. H. (1980). The development of ocular dominance columns in normal and visually deprived monkeys. J. Comp. Neurol. 191, 1-51. doi: 10.1002/cne.901910102 
Levinson, J. N., and El-Husseini, A. (2005). Building excitatory and inhibitory synapses: balancing neuroligin partnerships. Neuron 48, 171-174. doi: 10.1016/j.neuron.2005.09.017

Li, P., Li, Y. H., and Han, T. Z. (2009). NR2A-containing NMDA receptors are required for LTP induction in rat dorsolateral striatum in vitro. Brain Res. 1274, 40-46. doi: 10.1016/j.brainres.2009.04.016

Lin, H., Huganir, R., and Liao, D. (2004). Temporal dynamics of NMDA receptorinduced changes in spine morphology and AMPA receptor recruitment to spines. Biochem. Biophys. Res. Commun. 316, 501-511. doi: 10.1016/j.bbrc. 2004.02.086

Lovinger, D. M. (2010). Neurotransmitter roles in synaptic modulation, plasticity and learning in the dorsal striatum. Neuropharmacology 58, 951-961. doi: 10.1016/j.neuropharm.2010.01.008

Lovinger, D. M., and Mathur, B. N. (2012). Endocannabinoids in striatal plasticity. Parkinsonism Relat. Disord. 18, S132-S134. doi: 10.1016/S1353-8020(11) 70041-4

Lu, W.-Y., Man, H.-Y., Ju, W., Trimble, W. S., MacDonald, J. F., and Wang, Y. T. (2001). Activation of synaptic NMDA receptors induces membrane insertion of new AMPA receptors and LTP in cultured hippocampal neurons. Neuron 29, 243-254. doi: 10.1016/s0896-6273(01)00194-5

Ma, T., Cheng, Y., Roltsch Hellard, E., Wang, X., Lu, J., Gao, X., et al. (2018). Bidirectional and long-lasting control of alcohol-seeking behavior by corticostriatal LTP and LTD. Nat. Neurosci. 21, 373-383. doi: 10.1038/s41593018-0081-9

Maletic-Savatic, M., and Malinow, R. (1998). Calcium-evoked dendritic exocytosis in cultured hippocampal neurons. Part I: trans-golgi networkderived organelles undergo regulated exocytosis. J. Neurosci. 18, 6803-6813. doi: 10.1523/JNEUROSCI.18-17-06803.1998

Malinow, R., and Malenka, R. C. (2002). AMPA receptor trafficking and synaptic plasticity. Ann. Rev. Neurosci. 25, 103-126. doi: 10.1146/annurev.neuro.25. 112701.142758

Matikainen-Ankney, B. A., Kezunovic, N., Menard, C., Flanigan, M., Zhong, Y., Russo, S. J., et al. (2018). Parkinson's disease-linked LRRK2-G2019S mutation alters synaptic plasticity and promotes resilience to chronic social stress in young adulthood. J. Neurosci. 38, 9700-9711. doi: 10.1523/JNEUROSCI.145718.2018

Matsuo, N., Reijmers, L., and Mayford, M. (2008). Spine-type-specific recruitment of newly synthesized AMPA receptors with learning. Science 319, 1104-1107. doi: 10.1126/science.1149967

Matsuzaki, M., Honkura, N., Ellis-Davies, G. C. R., and Kasai, H. (2004). Structural basis of long-term potentiation in single dendritic spines. Nature 429, 761-766. doi: $10.1038 /$ nature02617

McKinney, R. A., Capogna, M., Dürr, R., Gähwiler, B. H., and Thompson, S. M. (1999). Miniature synaptic events maintain dendritic spines via AMPA receptor activation. Nat. Neurosci. 2, 44-49. doi: 10.1038/4548

McNeill, T. H., Brown, S. A., Rafols, J. A., and Shoulson, I. (1988). Atrophy of medium spiny I striatal dendrites in advanced Parkinson's disease. Brain Res. 455, 148-152. doi: 10.1016/0006-8993(88)90124-2

Milnerwood, A. J., Kaufman, A. M., Sepers, M. D., Gladding, C. M., Zhang, L., Wang, L., et al. (2012). Mitigation of augmented extrasynaptic NMDAR signaling and apoptosis in cortico-striatal co-cultures from Huntington's disease mice. Neurobiol. Dis. 48, 40-51. doi: 10.1016/j.nbd.2012.05.013

Milnerwood, A. J., and Raymond, L. A. (2010). Early synaptic pathophysiology in neurodegeneration: insights from Huntington's disease. Trends Neurosci. 33, 513-523. doi: 10.1016/j.tins.2010.08.002

Nägerl, U. V., Eberhorn, N., Cambridge, S. B., and Bonhoeffer, T. (2004). Bidirectional activity-dependent morphological plasticity in hippocampal neurons. Neuron 44, 759-767. doi: 10.1055/s-0040-1718441

O’Brien, R. J., Kamboj, S., Ehlers, M. D., Rosen, K. R., Fischbach, G. D., and Huganir, R. L. (1998). Activity-dependent modulation of synaptic AMPA receptor accumulation. Neuron 21, 1067-1078. doi: 10.1088/1361-6528/ abe154

Oh, W. C., Hill, T. C., and Zito, K. (2013). Synapse-specific and size-dependent mechanisms of spine structural plasticity accompanying synaptic weakening. Proc. Natl. Acad. Sci. U S A 110, E305-E312. doi: 10.1073/pnas.1214 705110

Okamoto, K. I., Nagai, T., Miyawaki, A., and Hayashi, Y. (2004). Rapid and persistent modulation of actin dynamics regulates postsynaptic reorganization underlying bidirectional plasticity. Nat. Neurosci. 7, 1104-1112. doi: $10.1038 / \mathrm{nn} 1311$

Okawa, H., Hoon, M., Yoshimatsu, T., Della Santina, L., and Wong, R. O. L. (2014). Illuminating the multifaceted roles of neurotransmission in shaping neuronal circuitry. Neuron 83, 1303-1318. doi: 10.1016/j.neuron.2014.08.029

Papa, M., and Segal, M. (1996). Morphological plasticity in dendritic spines of cultured hippocampal neurons. Neuroscience 71, 1005-1011. doi: 10.1016/0306-4522(95)00490-4

Paraskevopoulou, F., Herman, M. A., and Rosenmund, C. (2019). Glutamatergic innervation onto striatal neurons potentiates GABAergic synaptic output. J. Neurosci. 39, 4448-4460. doi: 10.1523/JNEUROSCI.2630-18.2019

Park, P., Kang, H., Sanderson, T. M., Bortolotto, Z. A., Georgiou, J., Zhuo, M., et al. (2018). The role of calcium-permeable AMPARs in long-term potentiation at principal neurons in the rodent hippocampus. Front. Synaptic Neurosci. 10, 1-11. doi: 10.3389/fnsyn.2018.00042

Park, H., Popescu, A., and Poo, M. (2014). Essential role of presynaptic NMDA receptors in activity-dependent BDNF secretion and corticostriatal LTP. Neuron 84, 1009-1022. doi: 10.1016/j.neuron.2014.10.045

Park, M., Salgado, J. M., Ostroff, L., Helton, T. D., Robinson, C. G., Harris, K. M., et al. (2006). Plasticity-induced growth of dendritic spines by exocytic trafficking from recycling endosomes. Neuron 52, 817-830. doi: 10.1016/j. neuron.2006.09.040

Penrod, R. D., Campagna, J., Panneck, T., Preese, L., and Lanier, L. M. (2015). The presence of cortical neurons in striatal-cortical co-cultures alters the effects of dopamine and BDNF on medium spiny neuron dendritic development. Front. Cell. Neurosci. 9, 1-14. doi: 10.3389/fncel.2015.00269

Penzes, P., Cahill, M. E., Jones, K. A., Vanleeuwen, J., and Woolfrey, K. M. (2011). Dendritic spine pathology in neuropsychiatric disorders. Nat. Neurosci. 14 285-293. doi: 10.1038/nn.2741

Perrin, E., and Venance, L. (2019). Bridging the gap between striatal plasticity and learning. Curr. Opin. Neurobiol. 54, 104-112. doi: 10.1016/j.conb.2018.09.007

Portera-Cailliau, C., Pan, D. T., and Yuste, R. (2003). Activity-regulated dynamic behavior of early dendritic protrusions: evidence for different types of dendritic filopodia. J. Neurosci. 23, 7129-7142. doi: 10.1523/JNEUROSCI.23-18-07 129.2003

Ramirez, D. M. O., and Kavalali, E. T. (2011). Differential regulation of spontaneous and evoked neurotransmitter release at central synapses. Curr. Opin. Neurobiol. 21, 275-282. doi: 10.1016/j.conb.2011.01.007

Randall, F. E., Garcia-Munoz, M., Vickers, C., Schock, S. C., Staines, W. A., and Arbuthnott, G. W. (2011). The corticostriatal system in dissociated cell culture. Front. Syst. Neurosci. 5, 1-7. doi: 10.3389/fnsys.2011.00052

Rao, A., and Craig, A. M. (1997). Activity regulates the synaptic localization of the NMDA receptor in hippocampal neurons. Neuron 19, 801-812. doi: 10.1016/s0896-6273(00)80962-9

Reynolds, J. N. J., and Wickens, J. R. (2002). Dopamine-dependent plasticity of corticostriatal synapses. Neural Netw. 15, 507-521. doi: 10.1016/s08936080(02)00045-x

Richards, D. A., Mateos, J. M., Hugel, S., de Paola, V., Caroni, P., Gähwiler, B. H., et al. (2005). Glutamate induces the rapid formation of spine head protrusions in hippocampal slice cultures. Proc. Natl. Acad. Sci. U S A 102, 6166-6171. doi: 10.1073/pnas.0501881102

Rutherford, L. C., DeWan, A., Lauer, H. M., and Turrigiano, G. G. (1997). Brain-derived neurotrophic factor mediates the activity-dependent regulation of inhibition in neocortical cultures. J. Neurosci. 17, 4527-4535. doi: 10.1523/JNEUROSCI.17-12-04527.1997

Sabo, S. L., Gomes, R. A., and McAllister, A. K. (2006). Formation of presynaptic terminals at predefined sites along axons. J. Neurosci. 26, 10813-10825. doi: 10 1523/JNEUROSCI.2052-06.2006

Sala, C., and Segal, M. (2014). Dendritic spines: the locus of structural and functional plasticity. Physiol. Rev. 94, 141-188. doi: 10.1152/physrev.000 12.2013

Sanes, J. R., and Lichtman, J. W. (1999). Development of the vertebrate neuromuscular junction. Annu. Rev. Neurosci. 22, 389-442. doi: 10. 1146/annurev.neuro.22.1.389

Sando, R., Bushong, E., Zhu, Y., Huang, M., Considine, C., Phan, S., et al. (2017). Assembly of excitatory synapses in the absence of glutamatergic neurotransmission. Neuron 94, 312.e3-321.e3. doi: 10.1016/j.neuron.2017. 03.047 
Schilling, K., Dickinson, M. H., Connor, J. A., and Morgan, J. I. (1991). Electrical activity in cerebellar cultures determines Purkinje cell dendritic growth patterns. Neuron 7, 891-902. doi: 10.1016/0896-6273(91)90335-w

Segal, M., Greenberger, V., and Korkotian, E. (2003). Formation of dendritic spines in cultured striatal neurons depends on excitatory afferent activity. Eur. J. Neurosci. 17, 2573-2585. doi: 10.1046/j.1460-9568.2003.02696.x

Sergeeva, O. A., Doreulee, N., Chepkova, A. N., Kazmierczak, T., and Haas, H. L. (2007). Long-term depression of cortico-striatal synaptic transmission by DHPG depends on endocannabinoid release and nitric oxide synthesis. Eur. J. Neurosci. 26, 1889-1894. doi: 10.1111/j.1460-9568.2007.05815.x

Sharma, K., Fong, D. K., and Craig, A. M. (2006). Postsynaptic protein mobility in dendritic spines: long-term regulation by synaptic NMDA receptor activation. Mol. Cell. Neurosci. 31, 702-712. doi: 10.1016/j.mcn.2006.01.010

Shehadeh, J., Fernandes, H. B., Zeron Mullins, M. M., Graham, R. K., Leavitt, B. R., Hayden, M. R., et al. (2006). Striatal neuronal apoptosis is preferentially enhanced by NMDA receptor activation in YAC transgenic mouse model of Huntington disease. Neurobiol. Dis. 21, 392-403. doi: 10.1016/j.nbd.2005. 08.001

Shen, W., Flajolet, M., Greengard, P., and Surmeier, D. J. (2008). Dichotomous dopaminergic control of striatal synaptic plasticity. Science 321, 848-851. doi: $10.1126 /$ science. 1160575

Shi, S.-H., Hayashi, Y., Esteban, J. A., and Malinow, R. (2001). Subunit-specific rules governing AMPA receptor trafficking to synapses in hippocampal pyramidal neurons. Cell 105, 331-343. doi: 10.1016/s0092-8674(01) 00321-x

Sigler, A., Oh, W. C., Imig, C., Altas, B., Kawabe, H., Cooper, B. H., et al. (2017). Formation and maintenance of functional spines in the absence of presynaptic glutamate release. Neuron 94, 304.e4-311.e4. doi: 10.1016/j.neuron.2017.03.029

Smith, Y., Villalba, R. M., and Raju, D. V. (2009). Striatal spine plasticity in Parkinson's disease: pathological or not? Parkinsonism Relat. Disord. 15, S156-S161. doi: 10.1016/S1353-8020(09)70805-3

Soares, C., Lee, K. F., Nassrallah, W., and Beique, J. C. (2013). Differential subcellular targeting of glutamate receptor subtypes during homeostatic synaptic plasticity. J. Neurosci. 33, 13547-13559. doi: 10.1523/JNEUROSCI. 1873-13.2013

Spencer, J. P., and Murphy, K. P. S. J. (2000). Bi-directional changes in synaptic plasticity induced at corticostriatal synapses in vitro. Exp. Brain Res. 135, 497-503. doi: 10.1007/s002210000523

Stellwagen, D., and Malenka, R. C. (2006). Synaptic scaling mediated by glial TNFa. Nature 440, 1054-1059. doi: 10.1038/nature04671

Takada, N., Yanagawa, Y., and Komatsu, Y. (2005). Activity-dependent maturation of excitatory synaptic connections in solitary neuron cultures of mouse neocortex. Eur. J. Neurosci. 21, 422-430. doi: 10.1111/j.1460-9568.2005. 03881.x

Tanaka, J.-I., Horiike, Y., Matsuzaki, M., Miyazaki, T., Ellis-Davies, G. C. R., and Kasai, H. (2008). Protein synthesis and neurotrophin-dependent structural plasticity of single dendritic spines. Science 319, 1683-1687. doi: $10.1126 /$ science. 1152864

Tang, K.-C., Low, M. J., Grandy, D. K., and Lovinger, D. M. (2001). Dopaminedependent synaptic plasticity in striatum during in vivo development. Proc. Natl. Acad. Sci. U S A 98, 1255-1260. doi: 10.1073/pnas.031374698

Tepper, J. M., Sharpe, N. A., Koós, T. Z., and Trent, F. (1998). Postnatal development of the rat neostriatum: electrophysiological, light- and electronmicroscopic studies. Dev. Neurosci. 20, 125-145. doi: 10.1159/000017308

Thibault, D., Giguère, N., Loustalot, F., Bourque, M. J., Ducrot, C., El Mestikawy, S., et al. (2016). Homeostatic regulation of excitatory synapses on striatal medium spiny neurons expressing the D2 dopamine receptor. Brain Struct. Funct. 221, 2093-2107. doi: 10.1007/s00429-015-1029-4
Thibault, D., Loustalot, F., Fortin, G. M., Bourque, M. J., and Trudeau, L. É. (2013). Evaluation of D1 and D2 dopamine receptor segregation in the developing striatum using BAC transgenic mice. PLoS One 8, 1-8. doi: 10.1371/journal. pone.0067219

Tian, X., Kai, L., Hockberger, P. E., Wokosin, D. L., and Surmeier, D. J. (2010). MEF-2 regulates activity-dependent spine loss in striatopallidal medium spiny neurons. Mol. Cell. Neurosci. 44, 94-108. doi: 10.1016/j.mcn.2010. 01.012

Tritsch, N. X., and Sabatini, B. L. (2012). Dopaminergic modulation of synaptic transmission in cortex and striatum. Neuron 76, 33-50. doi: 10.1016/j.neuron. 2012.09.023

Turrigiano, G. (2011). Too many cooks? Intrinsic and synaptic homeostatic mechanisms in cortical circuit refinement. Ann. Rev. Neurosci. 34, 89-103. doi: 10.1146/annurev-neuro-060909-153238

Turrigiano, G. G., Leslie, K. R., Desai, N. S., Rutherford, L. C., and Nelson, S. B. (1998). Activity-dependent scaling of quantal amplitude in neocortical neurons. Nature 391, 892-896. doi: 10.1038/36103

Villalba, R. M., and Smith, Y. (2013). Differential striatal spine pathology in Parkinson's disease and cocaine addiction: a key role of dopamine? Neuroscience 251, 2-20. doi: 10.1016/j.neuroscience.2013. 07.011

Volta, M., Beccano-Kelly, D. A., Paschall, S. A., Cataldi, S., MacIsaac, S. E., Kuhlmann, N., et al. (2017). Initial elevations in glutamate and dopamine neurotransmission decline with age, as does exploratory behavior, in LRRK2 G2019S knock-in mice. eLife 6:e28377. doi: 10.7554/eLife. 28377

Wang, Z., Kai, L., Day, M., Ronesi, J., Yin, H. H., Ding, J., et al. (2006). Dopaminergic control of corticostriatal long-term synaptic depression in medium spiny neurons is mediated by cholinergic interneurons. Neuron 50 , 443-452. doi: 10.1016/j.neuron.2006.04.010

Wierenga, C. J., Walsh, M. F., and Turrigiano, G. G. (2006). Temporal regulation of the expression locus of homeostatic plasticity. J. Neurophysiol. 96, 2127-2133. doi: 10.1152/jn.00107.2006

Yang, Y., Wang, X., Frerking, M., and Zhou, Q. (2008). Spine expansion and stabilization associated with long-term potentiation. J. Neurosci. 28, 5740-5751. doi: 10.1523/JNEUROSCI.3998-07.2008

Yoshihara, Y., De Roo, M., and Muller, D. (2009). Dendritic spine formation and stabilization. Curr. Opin. Neurobiol. 19, 146-153. doi: 10.1016/j.conb.2009. 05.013

Zhao, C., Dreosti, E., and Lagnado, L. (2011). Homeostatic synaptic plasticity through changes in presynaptic calcium influx. J. Neurosci. 31, 7492-7496. doi: 10.1523/JNEUROSCI.6636-10.2011

Zhou, Q., Homma, K. J., and Poo, M. M. (2004). Shrinkage of dendritic spines associated with long-term depression of hippocampal synapses. TL - 44. Neuron 44, 749-757. doi: 10.1016/j.neuron.2004. 11.011

Conflict of Interest: The authors declare that the research was conducted in the absence of any commercial or financial relationships that could be construed as a potential conflict of interest.

Copyright (c) 2021 Kuhlmann, Wagner Valladolid, Quesada-Ramírez, Farrer and Milnerwood. This is an open-access article distributed under the terms of the Creative Commons Attribution License (CC BY). The use, distribution or reproduction in other forums is permitted, provided the original author(s) and the copyright owner(s) are credited and that the original publication in this journal is cited, in accordance with accepted academic practice. No use, distribution or reproduction is permitted which does not comply with these terms. 